\title{
Analytical solutions for the hygro-thermo-mechanical bending of FG beams using a new fifth order shear and normal deformation theory
}

\author{
S. M. Ghumare ${ }^{a, *}$, A. S. Sayyad ${ }^{a}$ \\ ${ }^{a}$ Department of Civil Engineering, Sanjivani College of Engineering, Savitribai Phule Pune University, Kopargaon-423603, \\ Maharashtra state, India \\ Received 15 January 2020; accepted 11 June 2020
}

\begin{abstract}
A new analytical solution is presented for functionally graded (FG) beams to investigate the bending behaviour under the hygro-thermo-mechanical loading using a new fifth order shear and normal deformation theory (FOSNDT). The material properties of the FG beam are varied along the thickness direction according to the power law index. In the present theory, a polynomial shape function is expanded up to fifth-order in terms of thickness coordinate to consider the effects of transverse shear and normal deformations. The present theory is free from the shear correction factor. Using the Navier's solution technique the closed-form solution is obtained for simply supported FG beams. All the results are presented in non-dimensional form and validated it by developing the classical beam theory (CBT), first order shear deformation theory (FSDT by Mindlin) and third order shear deformation theory (TSDT by Reddy) considering the hygro-thermo-mechanical loading effects which is mostly missing in the literature. It is noticed that the presented FOSNDT is very simple and accurate to predict the bending behaviour of FG beams under linear and non-linear hygro-thermo-mechanical loadings.
\end{abstract}

(c) 2020 University of West Bohemia. All rights reserved.

Keywords: FG beam, transverse shear deformation, transverse normal deformation, bending, thermal stresses, hygro-thermo-mechanical loading

\section{Introduction}

The composites are usually a mixture of two or more than two materials in combination. The materials having the proper and significant chemical properties are normally chosen to get the typical mixture and required strength. Functionally graded materials (FGMs) are similar kinds of composite material having unique characteristics. Nowadays, FG materials are used in many engineering and structural applications because of its novel properties. The greatest advantage of FG material is flexibility in design, which makes it more suitable to grade the material properties in any specific direction as per requirement. Currently, the main focus of scientists and researchers is to suggest and introduce a new type of material which is light in weight, suits for any environmental conditions, should be reliable in service performance and able to minimize the size of structural elements. The available materials are not much suitable for all the engineering applications, hence the FG beams are used in main structural components of aerospace vehicles, defense sector units, shipbuilding industries, seashore structures, many automobile and chemical industries. In fact, the beams made from FG materials are very strong to resist the thermal stresses produced in spacecrafts and aircrafts operations. FG beams are even most suitable to resist hygro stresses generally developed in seashore and marine structures located in humid environment.

\footnotetext{
*Corresponding author. Tel.: +91992 31040 11, e-mail: smghumare@ rediffmail.com. https://doi.org/10.24132/acm.2020.580
} 
Hence, before using FG beam for important engineering applications, it is absolutely necessary to study the bending behaviour of FG beam under hygro-thermo-mechanical loading. Laminated composites may fail due to delamination failure or stress concentration at the layer interfaces during the life span. To overcome the delamination failure one can use FG material which is free from layers and the properties of such a material can be tailored in specific direction using a power law index. The ceramic and metal are the two major constituents used to form the FG materials. The ceramic constituents are having low thermal conductivity and good for wearing resistance, whereas the metals having good ductile performance, which sustains the deformations and can prevent the fractures caused due to stress. Looking into current scenario, the present research is an attempt towards fulfillment of the industry demands by developing the new solution technique to analyze important weight sensitive structures where safety and accuracy is on top propriety.

The several analytical techniques, numerical methods and few elasticity solutions are available in the literature which is addressed by various researchers across the globe, to study the static and dynamic response of the FG beams under mechanical and thermo-mechanical loading. But there is acute shortage of literature on the FG beams subjected to hygro-thermo-mechanical loadings. Hence, the main aim of this study is to present the bending analysis of FG beams considering the linear and non-linear variations of hygro-thermo-mechanical loadings. In this study, non-linearity is related to loadings and not a geometric.

The CBT (classical beam theory) which is developed by Euler-Bernoulli ignores the effect of shear deformation. The CBT is a simple beam theory used for the analysis of thin beams hence it not recommended for the analysis of thick beams due to neglect of the shear deformation effect. In the thick beam analysis, CBT underestimates the stresses and deformations. In 1921, Timoshenko developed the FSDT which assumes the first order variation in the axial displacement. But this theory needs the problem dependent shear correction factor to address the correct behaviour shear deformation. FSDT also fails to satisfy the zero transverse shear stress conditions at the top and bottom surfaces of the beam. To address the deficiencies of CBT and FDST, many researchers are trying to develop the new kinds of higher order theories.

During the space plane project in 1984, Japanese scientists introduced the FG materials to resist the ultra-high temperature. The FG sheets tested under high temperature fluctuations across the thin cross-sectional thickness. The use and applications of the FG materials can be found in the literature by Koizumi [59,60], Muller et al. [69], Birman and Byrd [25], etc. The detailed information on the FG beams and plates is available in the review articles published by Jha et al. [53], Swaminathan et al. [90], Swaminathan and Sangeeta [91], Sayyad and Ghugal $[85,87,88]$. This reviews given the good insights over the available literature which influences the many researchers to extend their efforts to analyze the FG beams under linear and non-linear hygro-thermo-mechanical loading which is very rarely addressed in the literature. Sankar [80], Ding et al. [38], Zhong and Yu [99], Daouadji et al. [36], Chu et al. [31], Ying et al. [95] and Xu et al. [94] presented an elasticity solution for the FG beams. The 3-D elasticity solutions are analytically very complicated, cumbersome and difficult to solve. Therefore, researchers are taking interest to propose a new analytical techniques and numerical methods for the FG beams in which the numerical results are closer to the exact solutions obtained by elasticity solutions.

Kadoli et al. [54], Sayyad and Ghugal [83], Reddy [79], Benatta [10], Li et al [62], Pendhari et al. [77], Giunta et al. [47, 49], Thai and Vo [92], Li and Batra [61], Nguyen et al. [74], Bourada et al. [26], Menna et al. [67], Mohanty [68], Pandey and Parashar [76] and Hadji et al. [52], Adim et al. [8], Daouadji and Adim [33,34], Daouadji [32], Benferhat et al. [13], Adim 
and Daouadji [4] have addressed the bending response of the FG beam using various kinds of higher order shear deformation theories (HSDTs). Zenkour [96] studied the behaviour of laminated and sandwich beam considering the transverse normal effect using HSDT. Sayyad and Ghugal [84] developed the analytical solutions for the bending of FG beam using different boundary conditions. Chakraborty et al. [27], Frikha et al. [42], Kahya and Turan [55], Kim and Paulino [58], Kant and Gupta [56] and Filippi et al. [41] analyzed the FG beams using finite element approach. Benferhat et al. [11, 12, 14, 16, 17], Daouadji et al. [37], Hadji et al. [50] studied the porosity effect on the bending of FG beams and plates to investigate the normal and shear interfacial stresses.

Daouadji et al. [35], Chergui et al. [29], Rabahi et al. [78] presented the numerical and experimental results to study the flexural behaviour of steel and RC beams strengthened by laminates. Benhenni et al. [18-21], Benferhat [15], Bensattalah et al. [22,23], Hadji et al. [51], Adim et al. [7] reported the free vibration response for the FG, laminated beams and plates. Adim et al. [5, 6], Bensattalah et al. [24], Khalifa [57] examined the buckling response of FG and laminated composite plate using refined HSDTs. Abdelhak et al. [1,2], Chaded et al. [28] developed an analytical solution for sandwich FG plate composed of FG face sheets and isotropic homogeneous core.

The beam made from FG materials are most suitable in thermal environments due to low thermal conductivity of ceramic materials. The response of the FG beam using different models under thermal and thermo-mechanical load is reported by a few researchers like Aboudi et al. [3], Chin and Chen [30], Giunta et al. [48], Megharbel [66], Sankar and Tzeng [81], etc. The effect of non-linear thermo-mechanical loads on bending behaviour of FG beams is reported by Shen [89], Ma and Lee [63,64], Ma and Wang [65], Esfahani et al. [39], Arbind et al. [9], Nirmala and Upadhyay [100], etc. Toudehdehghan et al. [82] studied the effect of thermal environment on FG coated beams using clamped-clamped end condition. Zhau et al. [82] and Sator et al. [75] used the meshless method to address the thermal effects.

The hygro-thermo-mechanical analysis for the FG plate is studied by Zidi et al. [101], Zenkour et al. [97], Daouadji et al. [32], Zenkour and Radwan [86] and Sayyad and Ghugal [98] using different higher order theories and mathematical approaches. But the given literature is limited to only for FG plates; there is acute shortage of literature on FG beams subjected to non-linear hygro-thermo-mechanical loads. Recently, a fifth order theory has been used by Ghumare and Sayyad [43-46] for the analysis of FG beams and plates and also by Naik and Sayyad [70-73] for the analysis of laminated composite beams and plates subjected to mechanical and thermo-mechanical loadings.

The main focus of the present study is to investigate the bending behaviour of FG beam using fifth order shear and normal deformation theory under the non-linear hygro-thermo-mechanical loadings. In the present theory, a polynomial shape functions are expanded to fifth-order in terms of the thickness coordinates. This theory includes the effect of thickness stretching. The theory involves only six independent field variables. This theory does not require shear correction factor. To obtain the closed-form solution, the Navier's solution technique is used for simply supported FG beam. Using power-law the material properties are graded. Using the principle of virtual work and the fundamental lemma of calculus, the variationally consistent governing equations and associated boundary conditions are evolved. To validate the present theory authors have developed CBT, FSDT and TSDT for the non-linear hygro-thermo-mechanical load and the dimensionless results are compared with these theories. It is found that the present FOSNDT is very simple and accurate to predict the bending response under the non-linear hygro-thermomechanical loadings. 


\section{Development of the present theory}

\subsection{Geometry of the FG Beam}

The dimensions and geometry of the FG beam used for the analytical solution are shown in Fig. 1. The $y$-directional width of the FG beam is assumed as unity. The top surface of the FG beam is made up of metal, whereas the bottom surface is made up of ceramic material.

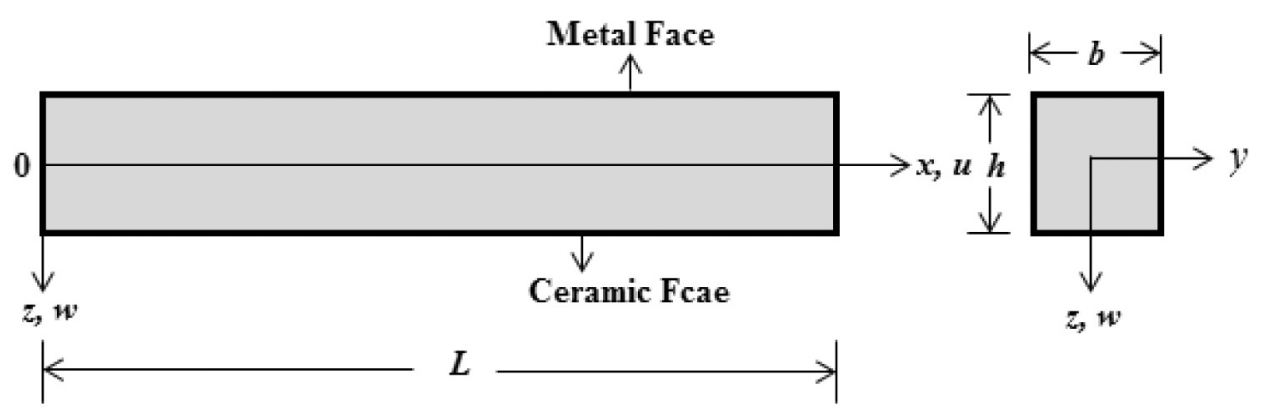

Fig. 1. Geometry of the FG beam

\subsection{Novelty and purpose of the present theory}

Looking at the current scenario, the present research is an attempt towards fulfillment of the industry demands and development in new solution technique to analyze important weight sensitive engineering structures.

1. It is observed that the numerous literature is available for mechanical loading $[4,8,10$, $13,26,27,31-34,36,38,41,42,47,49,52,54-56,58,61,62,67,68,74,76,77,79,80,83$, $84,92,94-96,99]$ and even for the thermo-mechanical loading $[3,9,30,39,48,63-66,75$, $81,82,89,93,100]$ for the FG beams, but there is acute shortage of literature on FG beam subjected to non-linear hygro-thermo-mechanical loading which is the main focus of the present study.

2. The present theory falls under polynomial type which is computationally very simpler than non-polynomial type beam theories which are mathematically complicated, tedious and more cumbersome.

3. For the accurate structural analysis of composite beams under hygro-thermal loading, considering the thickness coordinate up to third-order polynomial is not sufficient. Therefore, in the present theory thickness coordinate is expanded up to fifth-order polynomial to get the accurate displacements and stresses.

4. Transverse normal stress/strain plays an important role in the modeling of thick beams which is neglected by many theories available in the literature. The present theory considers the effects of both transverse shear and normal deformations.

5. The displacement field of the theory enforces the realistic variation of the transverse shear stresses (parabolic) across the thickness of the FG beam.

6. To grade the material a simple mixture rule, i.e. power-law is used.

\section{The development of the present fifth order theory}

\subsection{The displacement field}

The displacement field of the present theory is as follows:

$$
\begin{aligned}
u(x, z) & =u_{0}(x)-z \frac{\partial w_{0}}{\partial x}+F_{1} \phi_{x}(x)+F_{2} \psi_{x}(x), \\
w(x, z) & =w_{0}(x)+F_{1}^{\prime} \phi_{z}(x)+F_{2}^{\prime} \psi_{z}(x),
\end{aligned}
$$


where, $u$ and $w$ are the axial and transverse displacement of any point on the FG beam. $u_{0}$ and $w_{0}$ are the $x$ and $z$-directional displacements of any point on the neutral surface of the FG beam. The terms $\phi_{x}$ and $\psi_{x}$ are the shear slopes associated with the transverse shear deformation, whereas $\phi_{z}$ and $\psi_{z}$ are the shear slopes associated with the transverse normal deformations.

Using the theory of elasticity, the strain components are obtained:

$$
\begin{aligned}
\varepsilon_{x} & =\varepsilon_{x}^{0}+z k_{x}^{b}+F_{1} \varepsilon_{x}^{1}+F_{2} \varepsilon_{x}^{2}, \\
\varepsilon_{z} & =F_{1}^{\prime \prime} \phi_{z}+F_{2}^{\prime \prime} \psi_{z}, \\
\gamma_{x z} & =F_{1}^{\prime} \gamma_{x z}^{s_{1}}+F_{2}^{\prime} \gamma_{x z}^{s_{2}},
\end{aligned}
$$

where

$$
\begin{aligned}
\varepsilon_{x}^{0} & =\frac{\partial u_{0}}{\partial x}, \quad k_{x}^{b}=-\frac{\partial^{2} w_{0}}{\partial x^{2}}, \quad \varepsilon_{x}^{1}=\frac{\partial \phi_{x}}{\partial x}, \quad \varepsilon_{x}^{2}=\frac{\partial \psi_{x}}{\partial x} \\
\gamma_{x z}^{s_{1}} & =\left(\phi_{x}+\frac{\partial \phi_{z}}{\partial x}\right), \quad \gamma_{x z}^{s_{2}}=\left(\psi_{x}+\frac{\partial \psi_{z}}{\partial x}\right) \\
F_{1} & =\left(1-4 / 3 z^{3} / h^{2}\right), \quad F_{2}=\left(1-16 / 5 z^{5} / h^{4}\right), \\
F_{1}^{\prime} & =\left(1-4 z^{2} / h^{2}\right), \quad F_{2}^{\prime}=\left(1-16 z^{4} / h^{4}\right), \\
F_{1}^{\prime \prime} & =-\frac{8 z}{h^{2}}, \quad F_{2}^{\prime \prime}=-\frac{64 z^{3}}{h^{4}}
\end{aligned}
$$

\subsection{The power-law for material gradation}

The power law is used to change the volume fraction of the constituent materials continuously along the thickness direction. The power-law is stated as

$$
E(z)=E_{m}+\left(E_{c}-E_{m}\right)(0.5+z / h)^{p}
$$

where $E$ represents the elasticity modulus. Subscripts $m$ and $c$ represent the metal and ceramic constituent's, respectively, $p$ represents the power law index. The value of $p$ is equal to zero represents the fully ceramic phase, whereas $p$ equal to infinity represents a fully metallic phase. The effect of variation of the Poisson's ratio $\nu$ on the bending response of FG beam is very small, hence the Poisson's ratio is assumed as constant. The variation of thermal and moisture loads are also assumed to vary according to power-law index through the thickness of a FG beam. Fig. 2 shows the variation of volume fractions across the thickness of the FG beam.

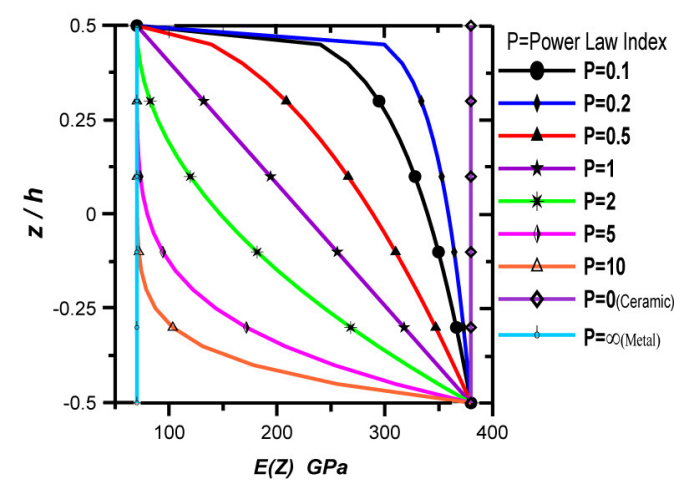

Fig. 2. Variation of elastic modulus across the FG beam 


\subsection{The constitutive law for the FG beam}

The FG beam follows the linear constitutive relations at a point can be written as follows,

$$
\left\{\begin{array}{c}
\sigma_{x} \\
\sigma_{z} \\
\tau_{x z}
\end{array}\right\}=\left[\begin{array}{ccc}
Q_{11}(z) & Q_{13}(z) & 0 \\
Q_{13}(z) & Q_{33}(z) & 0 \\
0 & 0 & Q_{55}(z)
\end{array}\right]\left\{\begin{array}{c}
\varepsilon_{x}-\alpha_{x} \Delta T-\beta_{x} \Delta C \\
\varepsilon_{z}-\alpha_{z} \Delta T-\beta_{z} \Delta C \\
\gamma_{x z}
\end{array}\right\},
$$

where

$$
Q_{11}(z)=Q_{33}(z)=\frac{E(z)}{1-\nu^{2}}, \quad Q_{13}(z)=\frac{\nu E(z)}{1-\nu^{2}}, \quad Q_{55}(z)=\frac{E(z)}{2(1+\nu)},
$$

$\alpha_{x}, \alpha_{z}$ and $\beta_{x}, \beta_{z}$ represent the coefficients of thermal and moisture concentration coefficients, respectively. The temperature and moisture variation profiles are assumed as follows,

$$
\begin{aligned}
& \Delta T(x, z)=T_{0}+\frac{z}{h} T_{1}+\frac{F_{1}}{h} T_{2}+\frac{F_{2}}{h} T_{3}, \\
& \Delta C(x, z)=C_{0}+\frac{z}{h} C_{1}+\frac{F_{1}}{h} C_{2}+\frac{F_{2}}{h} C_{3},
\end{aligned}
$$

where $T_{0}$ represents constant temperature, $T_{1}$ represents a linear temperature variation, $T_{2}$ and $T_{3}$ represents non-linear temperature variation (see Fig. 3). The similar meaning and variations are assumed for moisture concentration terms $C_{0}, C_{1}, C_{2}, C_{3}$.

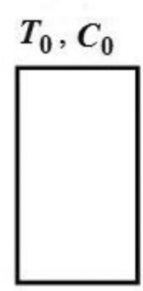

Constant

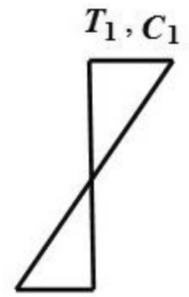

Linear

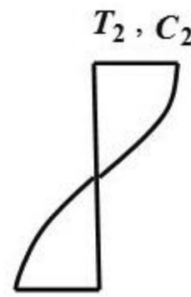

Non-Linear

Fig. 3. Temprature variation profile across the thickness of FG beam

\subsection{The governing equations}

The six variationally consistent governing equations are obtained using the principle of virtual displacement which equates external and internal work:

$$
\int_{0}^{L} \int_{-h / 2}^{h / 2}\left(\sigma_{x} \delta \varepsilon_{x}+\sigma_{z} \delta \varepsilon_{z}+\tau_{x z} \delta \gamma_{x z}\right) \mathrm{d} z \mathrm{~d} x=\int_{0}^{L}\left[q(x) \delta w_{0}\right] \mathrm{d} x .
$$

Substituing strains from (2) and stresses from (5) into the equation (8), one will get

$$
\begin{gathered}
\int_{0}^{L}\left(N_{x} \frac{\partial \delta u_{0}}{\partial x}-M_{x}^{b} \frac{\partial^{2} \delta w_{0}}{\partial x^{2}}+M_{x}^{S_{1}} \frac{\partial \delta \phi_{x}}{\partial x}+M_{x}^{S_{2}} \frac{\partial \delta \psi_{x}}{\partial x}+\right. \\
Q_{z}^{S_{1}} \delta \phi_{z}+Q_{z}^{S_{2}} \delta \psi_{z}+Q_{x z}^{1} \delta \phi_{x}+ \\
\left.Q_{x z}^{1} \frac{\partial \delta \phi_{z}}{\partial x}+Q_{x z}^{2} \delta \psi_{x}+Q_{x z}^{2} \frac{\partial \delta \psi_{z}}{\partial x}\right) \mathrm{d} x=\int_{0}^{L}\left[q(x) \delta w_{0}\right] \mathrm{d} x
\end{gathered}
$$


S. M. Ghumare et al. / Applied and Computational Mechanics 14 (2020) 5-30

$$
\begin{aligned}
{\left[N_{x}, M_{x}^{b}, M_{x}^{S_{1}}, M_{x}^{S_{2}}\right] } & =\int_{-h / 2}^{h / 2} \sigma_{x}\left[1, z, F_{1}, F_{2}\right] \mathrm{d} z \\
{\left[Q_{x z}^{1}, Q_{x z}^{2}\right] } & =\int_{-h / 2}^{h / 2} \tau_{x z}\left[F_{1}^{\prime}, F_{2}^{\prime}\right] \mathrm{d} z \\
{\left[Q_{z}^{S_{1}}, Q_{z}^{S_{2}}\right] } & =\int_{-h / 2}^{h / 2} \sigma_{z}\left[F_{1}^{\prime \prime}, F_{2}^{\prime \prime}\right] \mathrm{d} z
\end{aligned}
$$

where $N_{x}$ is the resultant axial force, $M_{X}^{b}$ represents the resultant moment due to bending, $M_{X}^{S_{1}}$, $M_{X}^{S_{2}}$ are the resultant moments due to shear deformation and $Q_{x z}^{1}, Q_{x z}^{2}, Q_{z}^{S_{1}}, Q_{z}^{S_{2}}$ denote the resultant shear forces.

The governing equations mentioned in (11)-(16) are obtained by integrating (11) by parts and setting the coefficients of $\delta u_{0}, \delta w_{0}, \delta \phi_{x}, \delta \psi_{x}, \delta \phi_{z}, \delta \psi_{z}$ and equating it to zero:

$\delta u_{0}$

$$
\begin{aligned}
& A_{11} \frac{\partial^{2} u_{0}}{\partial x^{2}}-B_{11} \frac{\partial^{3} w_{0}}{\partial x^{3}}+C_{11} \frac{\partial^{2} \phi_{x}}{\partial x^{2}}+D_{11} \frac{\partial^{2} \psi_{x}}{\partial x^{2}}+F_{13} \frac{\partial \phi_{z}}{\partial x}+H_{13} \frac{\partial \psi_{z}}{\partial x}- \\
& \left(A_{11}^{T}+A_{13}^{T}\right) \frac{\partial T_{0}}{\partial x}-\left(B_{11}^{T}+B_{13}^{T}\right) \frac{\partial T_{1}}{\partial x}-\left(C_{11}^{T}+C_{13}^{T}\right) \frac{\partial T_{2}}{\partial x}-\left(D_{11}^{T}+D_{13}^{T}\right) \frac{\partial T_{3}}{\partial x}- \\
& \left(A_{11}^{C}+A_{13}^{C}\right) \frac{\partial C_{0}}{\partial x}-\left(B_{11}^{C}+B_{13}^{C}\right) \frac{\partial C_{1}}{\partial x}-\left(C_{11}^{C}+C_{13}^{C}\right) \frac{\partial C_{2}}{\partial x}-\left(D_{11}^{C}+D_{13}^{C}\right) \frac{\partial C_{3}}{\partial x}=0
\end{aligned}
$$

$\delta w_{0}$ :

$$
\begin{gathered}
B_{11} \frac{\partial^{3} u_{0}}{\partial x^{2}}-A_{S 11} \frac{\partial^{4} w_{0}}{\partial x^{4}}+C_{S 11} \frac{\partial^{3} \phi_{x}}{\partial x^{3}}+D_{S 11} \frac{\partial^{3} \psi_{x}}{\partial x^{3}}+F_{S 13} \frac{\partial^{2} \phi_{Z}}{\partial x^{2}}+ \\
H_{S 13} \frac{\partial^{2} \psi_{Z}}{\partial x^{2}}-\left(B_{11}^{T}\right) \frac{\partial^{2} T_{0}}{\partial x^{2}}-\left(B_{13}^{T}\right) \frac{\partial^{2} T_{0}}{\partial x^{2}}-\left(A_{S 11}^{T}+A_{S 13}^{T}\right) \frac{\partial^{2} T_{1}}{\partial x^{2}}- \\
\left(C_{S 11}^{T}+C_{S 13}^{T}\right) \frac{\partial^{2} T_{2}}{\partial x^{2}}-\left(D_{S 11}^{T}+D_{S 13}^{T}\right) \frac{\partial^{2} T_{3}}{\partial x^{2}}-\left(B_{11}^{C}+B_{13}^{C}\right) \frac{\partial^{2} C_{0}}{\partial x^{2}}- \\
\left(A_{S 11}^{C}+A_{S 13}^{C}\right) \frac{\partial^{2} C_{1}}{\partial x^{2}}-\left(C_{S 11}^{C}+C_{S 13}^{C}\right) \frac{\partial^{2} C_{2}}{\partial x^{2}}-\left(D_{S 11}^{C}+D_{S 13}^{C}\right) \frac{\partial^{2} C_{3}}{\partial x^{2}}=-q,
\end{gathered}
$$

$\delta \phi_{x}: \quad C_{11} \frac{\partial^{2} u_{0}}{\partial x^{2}}-C_{S 11} \frac{\partial^{3} w_{0}}{\partial x^{3}}+C_{S S 111} \frac{\partial^{2} \phi_{x}}{\partial x^{2}}+C_{S S 211} \frac{\partial^{2} \psi_{x}}{\partial x^{2}}+F_{S S 13} \frac{\partial \phi_{Z}}{\partial x}+$

$$
\begin{gathered}
H_{S S 13} \frac{\partial \psi_{Z}}{\partial x}-F_{S S 155} \phi_{x}-F_{S S 155} \frac{d \phi_{x}}{\mathrm{~d} x}-H_{S S S 55}\left(\psi_{x}+\frac{\mathrm{d} \psi_{x}}{\mathrm{~d} x}\right)- \\
\left(C_{11}^{T}+C_{13}^{T}\right) \frac{\partial T_{0}}{\partial x}-\left(C_{S 11}^{T}+C_{S 13}^{T}\right) \frac{\partial T_{1}}{\partial x}-\left(C_{S S 111}^{T}+C_{S S 113}^{T}\right) \frac{\partial T_{2}}{\partial x}- \\
\left(C_{S S 211}^{T}-C_{S S 213}^{T}\right) \frac{\partial T_{3}}{\partial x}-\left(C_{11}^{C}+C_{13}^{C}\right) \frac{\partial C_{0}}{\partial x}-\left(C_{S 11}^{C}+C_{S 13}^{C}\right) \frac{\partial C_{1}}{\partial x}- \\
\left(C_{S S 111}^{C}+C_{S S 113}^{C}\right) \frac{\partial C_{2}}{\partial x}-\left(C_{S S 211}^{C}+C_{S S 213}^{C}\right) \frac{\partial C_{3}}{\partial x}=0,
\end{gathered}
$$

$\delta \psi_{x}: \quad D_{11} \frac{\partial^{2} u_{0}}{\partial x^{2}}-D_{S 11} \frac{\partial^{3} w_{0}}{\partial x^{3}}+C_{S S 211} \frac{\partial^{2} \phi_{x}}{\partial x^{2}}+D_{S S 211} \frac{\partial^{2} \psi_{x}}{\partial x^{2}}+F_{S S S 13} \frac{\partial \phi_{Z}}{\partial x}+$

$$
\begin{gathered}
H_{S S S 13} \frac{\partial \psi_{z}}{\partial x}-H_{S S S 55} \phi_{x}-H_{S S S 55} \frac{\partial \phi_{z}}{\partial x}-F_{S S 255}\left(\psi_{x}+\frac{\mathrm{d} \psi_{z}}{\mathrm{~d} x}\right)- \\
\left(D_{S 11}^{T}+D_{S 13}^{T}\right) \frac{\partial T_{1}}{\partial x}-\left(C_{S S 211}^{T}+C_{S S 213}^{T}\right) \frac{\partial T_{2}}{\partial x}-\left(D_{11}^{T}+D_{13}^{T}\right) \frac{\partial T_{0}}{\partial x}- \\
\left(D_{S S 211}^{T}+D_{S S 213}^{T}\right) \frac{\partial T_{3}}{\partial x}-\left(D_{11}^{C}+D_{13}^{C}\right) \frac{\partial C_{0}}{\partial x}-\left(D_{S 11}^{C}+D_{S 13}^{C}\right) \frac{\partial C_{1}}{\partial x}-
\end{gathered}
$$


S. M. Ghumare et al. / Applied and Computational Mechanics 14 (2020) 5-30

$$
\left(C_{S S 211}^{C}+C_{S S 213}^{C}\right) \frac{\partial C_{2}}{\partial x}-\left(D_{S S 211}^{C}+D_{S S 213}^{C}\right) \frac{\partial C_{3}}{\partial x}=0
$$

$\delta \phi_{z}: \quad-F_{13} \frac{\partial u_{0}}{\partial x}+F_{S 13} \frac{\partial^{2} w_{0}}{\partial x^{2}}-F_{S S 13} \frac{\partial \phi_{x}}{\partial x}-F_{S S S 13} \frac{\partial \psi_{x}}{\partial x}-F_{S S S 133} \phi_{Z}-$

$$
\begin{gathered}
F_{S S S 233} \psi_{z}-F_{S S 155} \frac{\partial \phi_{x}}{\partial x}-F_{S S 155} \frac{\partial^{2} \phi_{z}}{\partial x^{2}}+H_{S S S 55}\left(\frac{\partial \psi_{x}}{\partial x}+\frac{\partial^{2} \psi_{z}}{\partial x^{2}}\right)- \\
\left(F_{13}^{T}+F_{33}^{T}\right) T_{0}-\left(F_{S 13}^{T}+F_{S 33}^{T}\right) T_{1}-\left(F_{S S 113}^{T}+F_{S S 133}^{T}\right) T_{2}- \\
\left(F_{S S 213}^{T}+F_{S S 233}^{T}\right) T_{3}-\left(F_{13}^{C}+F_{33}^{C}\right) C_{0}-\left(F_{S 13}^{C}+F_{S 33}^{C}\right) C_{1}- \\
\left(F_{S S 113}^{C}+F_{S S 133}^{C}\right) C_{2}-\left(F_{S S 213}^{C}+F_{S S 233}^{C}\right) C_{3}=0,
\end{gathered}
$$

$\delta \psi_{z}: \quad-H_{13} \frac{\partial u_{0}}{\partial x}+H_{S 13} \frac{\partial^{2} w_{0}}{\partial x^{2}}-H_{S S 13} \frac{\partial \phi_{x}}{\partial x}-H_{S S S 13} \frac{\partial \psi_{x}}{\partial x}+F_{S S S 233} \phi_{Z}-$

$$
\begin{gathered}
H_{S S S 233} \psi_{z}+H_{S S S 55}\left(\frac{\partial \phi_{x}}{\partial x}+\frac{\partial^{2} \phi_{z}}{\partial x^{2}}\right)+F_{S S 255}\left(\frac{\partial \psi_{x}}{\partial x}+\frac{\partial^{2} \psi_{z}}{\partial x^{2}}\right)- \\
\left(H_{13}^{T}+H_{33}^{T}\right) T_{0}-\left(H_{S 13}^{T}+H_{S 33}^{T}\right) T_{1}-\left(H_{S S 113}^{T}+H_{S S 133}^{T}\right) T_{2}- \\
\left(H_{S S 213}^{T}+H_{S S 233}^{T}\right) T_{3}-\left(H_{13}^{C}+H_{33}^{C}\right) C_{0}-\left(H_{S 13}^{C}+H_{S 33}^{C}\right) C_{1}- \\
\left(H_{S S 113}^{C}+H_{S S 133}^{C}\right) C_{2}-\left(H_{S S 213}^{C}+H_{S S 233}^{C}\right) C_{3}=0 .
\end{gathered}
$$

The governing equations involve mechanical, thermal and moisture constants which are mentioned in (17)-(19) as follows:

Mechanical constants

$$
\begin{aligned}
& {\left[A_{i j}, B_{i j}, C_{i j}, D_{i j}\right] }=Q_{i j}(z) \int_{-h / 2}^{h / 2}\left[1, z, F_{1}, F_{2}\right] \mathrm{d} z \\
& {\left[A_{s i j}, C_{s i j}, D_{s i j}, F_{s i j}, H_{s i j}\right] }=Q_{i j}(z) \int_{-h / 2}^{h / 2} z\left[z, F_{1}, F_{2}, F_{1}^{\prime \prime}, F_{2}^{\prime \prime}\right] \mathrm{d} z \\
& {\left[C_{S S 1 i j}, C_{S S 2 i j}\right] }=Q_{i j}(z) \int_{-h / 2}^{h / 2} F_{1}\left[F_{1}, F_{2}\right] \mathrm{d} z \\
& {\left[D_{S S 2 i j}\right] }=Q_{i j}(z) \int_{-h / 2}^{h / 2} F_{2}^{2} \mathrm{~d} z, \quad\left[F_{i j}\right]=Q_{i j}(z) \int_{-h / 2}^{h / 2} F_{2}^{\prime \prime} \mathrm{d} z, \\
& {\left[H_{i j}\right] }=Q_{i j}(z) \int_{-h / 2}^{h / 2} F_{2}^{\prime \prime} \mathrm{d} z, \\
& {\left[F_{S S i j}, H_{S S i j}\right] }=Q_{i j}(z) \int_{-h / 2}^{h / 2} F_{1}\left[F_{1}^{\prime \prime}, F_{2}^{\prime \prime}\right] \mathrm{d} z, \\
& {\left[H_{S S S i j}, F_{S S 2 i j}\right] }=Q_{i j}(z) \int_{-h / 2}^{h / 2} F_{2}^{\prime}\left[F_{1}^{\prime}, F_{2}^{\prime}\right] \mathrm{d} z, \\
& {\left[F_{S S S i j}, H_{S S S i j}\right] }=Q_{i j}(z) \int_{-h / 2}^{h / 2} F_{2}\left[F_{1}^{\prime \prime}, F_{2}^{\prime \prime}\right] \mathrm{d} z, \\
& {\left[F_{S S 1 i j}\right] }=Q_{i j}(z) \int_{-h / 2}^{h / 2} F_{1}^{\prime} F_{1}^{\prime} \mathrm{d} z, \\
& {\left[F_{S S S 2 i j}, H_{S S S 2 i j}\right] }=Q_{i j}(z) \int_{-h / 2}^{h / 2} F_{2}^{\prime \prime}\left[F_{1}^{\prime \prime}, F_{2}^{\prime \prime}\right] \mathrm{d} z . \\
& {\left[F_{S S S 1 i j}\right]=Q_{i j}(z) \int_{-h / 2}^{h / 2} F_{1}^{\prime \prime} F_{1}^{\prime \prime} \mathrm{d} z }
\end{aligned}
$$


Thermal constants

$$
\begin{aligned}
{\left[A_{i j}^{T}, B_{i j}^{T}, C_{i j}^{T}, D_{i j}^{T}\right] } & =\alpha_{x} Q_{i j}(z) \int_{-h / 2}^{h / 2}\left[1, z, F_{1}, F_{2}\right] \mathrm{d} z \\
{\left[A_{s i j}^{T}, C_{s i j}^{T}, D_{s i j}^{T}, F_{s i j}^{T}, H_{s i j}^{T}\right] } & =\frac{\alpha_{x}}{h} Q_{i j}(z) \int_{-h / 2}^{h / 2} z\left[z, F_{1}, F_{2}, F_{1}^{\prime \prime}, F_{2}^{\prime \prime}\right] \mathrm{d} z \\
{\left[C_{S S 1 i j}^{T}, C_{S S 2 i j}^{T}\right] } & =\frac{\alpha_{x}}{h} Q_{i j}(z) \int_{-h / 2}^{h / 2} F_{1}\left[F_{1}, F_{2}\right] \mathrm{d} z \\
{\left[D_{S S 2 i j}^{T}\right] } & =\frac{\alpha_{x}}{h} Q_{i j}(z) \int_{-h / 2}^{h / 2} F_{2}^{2} \mathrm{~d} z, \\
{\left[F_{i j}^{T}\right] } & =\frac{\alpha_{x}}{h} Q_{i j}(z) \int_{-h / 2}^{h / 2} F_{1}^{\prime \prime} \mathrm{d} z \\
{\left[H_{i j}^{T}\right] } & =\frac{\alpha_{x}}{h} Q_{i j}(z) \int_{-h / 2}^{h / 2} F_{2}^{\prime \prime} \mathrm{d} z \\
{\left[F_{S S i j}^{T}, H_{S S i j}^{T}\right] } & =\frac{\alpha_{x}}{h} Q_{i j}(z) \int_{-h / 2}^{h / 2} F_{1}\left[F_{1}^{\prime \prime}, F_{2}^{\prime \prime}\right] \mathrm{d} z .
\end{aligned}
$$

Moisture constants

$$
\begin{aligned}
{\left[A_{i j}^{C}, B_{i j}^{C}, C_{i j}^{C}, D_{i j}^{C}\right] } & =\beta_{x} Q_{i j}(z) \int_{-h / 2}^{h / 2}\left[1, z, F_{1}, F_{2}\right] \mathrm{d} z \\
{\left[A_{s i j}^{C}, C_{s i j}^{C}, D_{s i j}^{C}, F_{s i j}^{C}, H_{s i j}^{C}\right] } & =\frac{\beta_{x}}{h} Q_{i j}(z) \int_{-h / 2}^{h / 2} z\left[z, F_{1}, F_{2}, F_{1}^{\prime \prime}, F_{2}^{\prime \prime}\right] \mathrm{d} z \\
{\left[C_{S S 1 i j}^{C}, C_{S S 2 i j}^{C}\right] } & =\frac{\beta_{x}}{h} Q_{i j}(z) \int_{-h / 2}^{h / 2} F_{1}\left[F_{1}, F_{2}\right] \mathrm{d} z, \\
{\left[D_{S S 2 i j}^{C}\right] } & =\frac{\beta_{x}}{h} Q_{i j}(z) \int_{-h / 2}^{h / 2} F_{2}^{2} \mathrm{~d} z, \quad\left[F_{i j}^{C}\right]=\frac{\beta_{x}}{h} Q_{i j}(z) \int_{-h / 2}^{h / 2} F_{1}^{\prime \prime} \mathrm{d} z, \\
{\left[H_{i j}^{C}\right] } & =\frac{\beta_{x}}{h} Q_{i j}(z) \int_{-h / 2}^{h / 2} F_{2}^{\prime \prime} \mathrm{d} z, \\
{\left[F_{S S i j}^{C}, H_{S S i j}^{C}\right] } & =\frac{\beta_{x}}{h} Q_{i j}(z) \int_{-h / 2}^{h / 2} F_{1}\left[F_{1}^{\prime \prime}, F_{2}^{\prime \prime}\right] \mathrm{d} z .
\end{aligned}
$$

\subsection{The boundary conditions}

The boundary conditions obtained at $x=0$ and $x=L$ are in the following form:

Either $N_{x}=0$ or $u_{0}$ is prescribed.

Either $M_{x}^{b}=0$ or $\partial w_{0} / \partial x$ is prescribed.

Either $\partial M_{x}^{b} / \partial x=0$ or $w_{b}$ is prescribed.

Either $M_{x}^{S_{1}}=0$ or $\phi_{x}$ is prescribed.

Either $M_{x}^{S_{2}}=0$ or $\psi_{x}$ is prescribed.

Either $Q_{x z}^{1}=0$ or $\phi_{z}$ is prescribed.

Either $Q_{x z}^{2}=0$ or $\psi_{z}$ is prescribed. 


\subsection{The analytical solution}

Using the Navier's solution technique, the analytical solution is obtained for the simply supported FG beam. The displacement variables are expressed in the single trigonometric series

$$
\begin{aligned}
& \left\{u_{0}, \phi_{x}, \psi_{x}\right\}=\sum_{m=1}^{\infty}\left\{u_{m}, \phi_{x m}, \psi_{x m}\right\} \cos \alpha x, \\
& \left\{w_{0}, \phi_{z}, \psi_{z}\right\}=\sum_{m=1}^{\infty}\left\{w_{m}, \phi_{z m}, \psi_{z m}\right\} \sin \alpha x,
\end{aligned}
$$

where $u_{m}, w_{m}, \phi_{x m}, \psi_{x m}, \phi_{z m}, \psi_{z m}$ are the unknown coefficients and $\alpha=m \pi / L$. The top surface of the beam is transversely loaded with $q(x)$ and load is expressed in a single trigonometric form as

$$
q(x)=\sum_{m=1}^{\infty} q_{m} \sin \alpha x,
$$

where

$$
q_{m}=q_{0} \quad \text { (sinusoidal load) }
$$

Using (30), the analytical solution is obtained by substituting the trigonometric form of $u_{0}, w_{0}$, $\phi_{x}, \psi_{x}, \phi_{z}, \psi_{z}$ and $q(x)$ from (27)-(29) into governing equations (11)-(16)

$$
[K]\{\Delta\}=\{f\}
$$

where $[K]$ represents the stiffness matrix, $\{f\}$ represents the force vector and $\{\Delta\}$ represents the unknowns coefficient vector. The elements of $[K],\{\Delta\}$ and $\{f\}$ are as follows:

$$
\begin{array}{lll}
K_{11}=-A_{11} \alpha^{2}, & K_{12}=B_{11} \alpha^{3}, & K_{13}=-C_{11} \alpha^{2}, \\
K_{14}=-D_{11} \alpha^{2}, & K_{15}=F_{13} \alpha, & K_{16}=H_{13} \alpha, \\
K_{22}=-A_{S 11} \alpha^{4}, & K_{23}=C_{S 11} \alpha^{3}, & K_{24}=D_{S 11} \alpha^{3}, \\
K_{25}=-F_{S 13} \alpha^{2}, & K_{26}=-H_{S 13} \alpha^{2}, & K_{33}=-\left(C_{S S 111} \alpha^{2}+F_{S S 155}\right), \\
K_{34}=-\left(C_{S S 211} \alpha^{2}+H_{S S S 55}\right), & K_{35}=\left(F_{S S 13}-F_{S S 155}\right) \alpha, \\
K_{36}=\left(H_{S S 13}-H_{S S S 55}\right) \alpha, & K_{44}=-\left(D_{S S 211} \alpha^{2}+F_{S S 255}\right), \\
K_{45}=\left(F_{S S S 13}-H_{S S S 55}\right) \alpha, & K_{46}=\left(H_{S S S 13}-F_{S S 255}\right) \alpha, \\
K_{55}=-\left(F_{S S 155} \alpha^{2}+F_{S S S 133}\right), & K_{56}=-\left(H_{S S S 155} \alpha^{2}+F_{S S S 233}\right), \\
K_{66}=-\left(F_{S S 255} \alpha^{2}+H_{S S S 233}\right), &
\end{array}
$$

symmetric elements:

$$
\begin{gathered}
K_{21}=K_{12}, K_{31}=K_{13}, K_{41}=K_{14}, K_{51}=K_{15}, K_{61}=K_{16}, K_{32}=K_{23}, \\
K_{42}=K_{24}, K_{43}=K_{34}, K_{52}=K_{25}, K_{62}=K_{26}, K_{53}=K_{35}, \\
K_{63}=K_{36}, K_{54}=K_{45}, K_{64}=K_{46}, K_{65}=K_{56}, \\
\{\Delta\}=\left\{u_{m}, w_{m}, \phi_{x m}, \psi_{x m}, \phi_{z m}, \psi_{z m}\right\}^{T}, \\
\{f\}=\left\{f_{1},-\left(f_{2}+q_{m}\right), f_{3}, f_{4}, f_{5}, f_{6}\right\}^{T},
\end{gathered}
$$


where

$$
\begin{aligned}
f_{1}= & -\left(A_{11}^{T}+A_{13}^{T}\right) t_{0} \alpha-\left(B_{11}^{T}+B_{13}^{T}\right) t_{1} \alpha-\left(C_{11}^{T}+C_{13}^{T}\right) t_{2} \alpha-\left(D_{11}^{T}+D_{13}^{T}\right) t_{3} \alpha- \\
& \left(A_{11}^{C}+A_{13}^{C}\right) C_{0} \alpha-\left(B_{11}^{C}+B_{13}^{C}\right) C_{1} \alpha-\left(C_{11}^{C}+C_{13}^{C}\right) C_{2} \alpha-\left(D_{11}^{C}+D_{13}^{C}\right) C_{3} \alpha, \\
f_{2}= & -q-\left(B_{11}^{T}+A_{13}^{T}\right) t_{0} \alpha^{2}-\left(A_{S 11}^{T}+A_{S 13}^{T}\right) t_{1} \alpha^{2}-\left(C_{S 11}^{T}+C_{S 13}^{T}\right) t_{2} \alpha^{2}- \\
& \left(D_{S 11}^{T}+D_{S 13}^{T}\right) t_{3} \alpha^{2}-\left(B_{11}^{C}+A_{13}^{C}\right) C_{0} \alpha^{2}-\left(A_{S 11}^{C}+A_{S 13}^{C}\right) C_{1} \alpha^{2}- \\
& \left(C_{S 11}^{C}+C_{S 13}^{C}\right) C_{2} \alpha^{2}-\left(D_{S 11}^{C}+D_{S 13}^{C}\right) C_{3} \alpha^{2}, \\
f_{3}= & -\left(C_{11}^{T}+C_{13}^{T}\right) t_{0} \alpha-\left(C_{S 11}^{T}+C_{S 13}^{T}\right) t_{1} \alpha-\left(C_{S S 111}^{T}+C_{S S 113}^{T}\right) t_{2} \alpha- \\
& \left(C_{S S 211}^{T}+C_{S S 213}^{T}\right) t_{3} \alpha-\left(C_{11}^{C}+C_{13}^{C}\right) C_{0} \alpha-\left(C_{S 11}^{C}+C_{S 13}^{C}\right) C_{1} \alpha- \\
& \left(C_{S S 111}^{C}+C_{S S 113}^{C}\right) C_{2} \alpha-\left(C_{S S 211}^{C}+C_{S S 213}^{C}\right) C_{3} \alpha, \\
f_{4}= & -\left(D_{11}^{T}+D_{13}^{T}\right) t_{0} \alpha-\left(D_{S 11}^{T}+D_{S 13}^{T}\right) t_{1} \alpha-\left(C_{S S 211}^{T}+C_{S S 213}^{T}\right) t_{2} \alpha- \\
& \left(D_{S S 111}^{T}+D_{S S 113}^{T}\right) t_{3} \alpha-\left(D_{11}^{C}+D_{13}^{C}\right) C_{0} \alpha-\left(D_{11}^{C}+D_{S 13}^{C}\right) C_{1} \alpha- \\
& \left(C_{S S 211}^{C}+C_{S S 213}^{C}\right) C_{2} \alpha-\left(D_{S S 111}^{C}+D_{S S 113}^{C}\right) C_{3} \alpha, \\
f_{5}= & -\left(F_{13}^{T}+F_{33}^{T}\right) t_{0}-\left(F_{S 13}^{T}+F_{S 33}^{T}\right) t_{1}-\left(F_{S S 113}^{T}+F_{S S 133}^{T}\right) t_{2}- \\
& \left(F_{S S 213}^{T}+F_{S S 233}^{T}\right) t_{3}-\left(F_{13}^{C}+F_{33}^{C}\right) C_{0}-\left(F_{S 13}^{C}+F_{S 33}^{C}\right) C_{1}- \\
& \left(F_{S S 113}^{C}+F_{S S 133}^{C}\right) C_{2}-\left(F_{S S 213}^{C}+F_{S S 233}^{C}\right) C_{3}, \\
f_{6}= & -\left(H_{13}^{T}+H_{33}^{T}\right) t_{0}-\left(H_{S 13}^{T}+H_{S 33}^{T}\right) t_{1}-\left(H_{S S 113}^{T}+H_{S S 133}^{T}\right) t_{2}- \\
& \left(H_{S S 213}^{T}+H_{S S 233}^{T}\right) t_{3}-\left(H_{13}^{C}+H_{33}^{C}\right) C_{0}-\left(H_{S 13}^{C}+H_{S 33}^{C}\right) C_{1}- \\
& \left(H_{S S 113}^{C}+H_{S S 133}^{C}\right) C_{2}-\left(H_{S S 213}^{C}+H_{S S 233}^{C}\right) C_{3} .
\end{aligned}
$$

\section{Illustrative examples, numerical results and discussion}

To verify the accuracy of the present theory, bending response under thermo-mechanical and hygro-thermo-mechanical loadings are presented and discussed in this section. For the analytical solution, the FG beam made of ceramic (Alumina: $E_{c}=380 \mathrm{GPa}, \nu_{c}=0.3$ ) and metal (Aluminum: $E_{m}=70 \mathrm{GPa}$ and $\left.\nu_{m}=0.3\right)$ is considered. Displacements and stresses are presented in the dimensionless form

$$
\begin{aligned}
\bar{w}\left(\frac{L}{2}, 0\right) & =\frac{100 E_{m} h^{3} w}{q_{0} L^{4}}, & \bar{u}\left(0,-\frac{h}{2}\right) & =\frac{100 E_{m} h^{3} u}{q_{0} L^{4}}, \\
\bar{\sigma}_{x}\left(\frac{L}{2}, \frac{h}{2}\right) & =\frac{h \sigma_{x}}{q_{0} L}, & \bar{\tau}_{x z}(0,0) & =\frac{h \tau_{x z}}{q_{0} L} .
\end{aligned}
$$

\subsection{Illustrative example}

To validate the present theory, the following examples are solved.

Example 1: Transverse displacements and stresses in FG beam under linear thermo-mechanical $\operatorname{load}\left(T_{1}=10, C_{1}=0, q_{0}=100\right)$.

Example 2: Transverse displacements and stresses in FG beam under non-linear thermomechanical load $\left(T_{1}=10, T_{2}=10, C_{1}=C_{2}=0, q_{0}=100\right)$.

Example 3: Transverse displacements and stresses in FG beam under linear hygro-thermomechanical load $\left(T_{1}=10, C_{1}=100, q_{0}=100\right)$.

Example 4: Transverse displacements and stresses in FG beam under non-linear hygro-thermomechanical load $\left(T_{1}=T_{2}=10, C_{1}=C_{2}=100, q_{0}=100\right)$. 


\subsection{Numerical results for the thermo-mechanical load and their discussion}

Table 1 shows the comparison of maximum dimensionless axial displacement $\bar{u}$, transverse deflection $\bar{w}$, normal stress $\bar{\sigma}_{x}$ and transverse shear stress $\bar{\tau}_{x z}$ for FG beams subjected to linear thermo-mechanical load with the power index $p$. The transverse and axial displacements obtained by the present theory are marginally less for ceramic and metal phases when $p=0, \infty$, whereas for $p=1,2,5,10$ the transverse and axial displacements are in close agreement with the other theories. It is observed that the displacement increases with an increase in power law index. This is due to the decrease in the stiffness of the FG beam. It is noted that neglecting the transverse shear deformation effect, the CBT underestimates the displacements. Also, it is important to observe that the numerical results for normal and transverse shear stresses are having a close agreement with other refined theories. Table 2 shows maximum values of dimensionless transverse deflection $\bar{w}$ under linear thermo-mechanical loads by varying the aspect ratio and power law index. As the aspect ratio increases, the dimensionless deflection found decreased, whereas deflection increases with power law. Table 3 shows the comparison of non-dimensional

Table 1. Comparison of axial displacement, transverse deflection, normal stress and transverse shear stress in FG beams subjected to linear thermal load $\left(L / h=10, T_{1}=10, q_{0}=100\right)$

\begin{tabular}{|cllrrrc|}
\hline$p$ & Theory & Model & $\bar{u}$ & $\bar{w}$ & $\bar{\sigma}_{x}$ & $\bar{\tau}_{x z}$ \\
\hline 0 & Present $\left(\varepsilon_{z} \neq 0\right)$ & FOSNDT & 0.4431 & 2.8214 & 7.6973 & 0.4768 \\
& Reddy [79] & PSDT & 0.4425 & 2.8186 & 7.4529 & 0.4773 \\
& Bernoulli-Euler [40] & EBT & 0.4353 & 2.8214 & 7.4268 & - \\
\hline 1 & Present $\left(\varepsilon_{z} \neq 0\right)$ & FOSNDT & 0.6431 & 5.5257 & 10.3162 & 0.5211 \\
& Reddy [79] & PSDT & 0.6402 & 5.2001 & 10.9227 & 0.5189 \\
& Bernoulli-Euler [40] & EBT & 0.6262 & 5.3079 & 10.6843 & - \\
\hline 2 & Present $\left(\varepsilon_{z} \neq 0\right)$ & FOSNDT & 0.7392 & 6.5080 & 12.5027 & 0.5461 \\
& Reddy [79] & PSDT & 0.7343 & 6.4961 & 12.5278 & 0.5487 \\
& Bernoulli-Euler [40] & EBT & 0.7209 & 6.7472 & 12.2995 & - \\
\hline 5 & Present $\left(\varepsilon_{z} \neq 0\right)$ & Present & 0.8609 & 7.7374 & 14.5250 & 0.5298 \\
& Reddy [79] & PSDT & 0.8542 & 7.7387 & 14.5720 & 0.5215 \\
& Bernoulli-Euler [40] & EBT & 0.8298 & 7.8119 & 14.1569 & - \\
\hline 10 & Present $\left(\varepsilon_{z} \neq 0\right)$ & FOSNDT & 1.0159 & 8.4799 & 17.1333 & 0.4245 \\
& Reddy [79] & PSDT & 0.9880 & 8.4827 & 17.2089 & 0.4142 \\
& Bernoulli-Euler [40] & EBT & 0.9814 & 8.3812 & 16.7431 & - \\
\hline$\infty$ & Present $\left(\varepsilon_{z} \neq 0\right)$ & FOSNDT & 2.0493 & 13.3446 & 21.3548 & 0.4771 \\
& Reddy [79] & PSDT & 2.0587 & 13.3451 & 21.3948 & 0.4769 \\
& Bernoulli-Euler [40] & EBT & 2.0475 & 13.0293 & 20.9943 & - \\
\hline
\end{tabular}

Table 2. Non-dimensional deflections $\bar{w}$ in FG beam subjected to linear thermo-mechanical load $\left(T_{1}=10\right)$

\begin{tabular}{|rrrrrrrr|}
\hline \multicolumn{7}{|c|}{ Aspect Ratio $(L / h)$} \\
\hline$p$ & \multicolumn{1}{c}{5} & \multicolumn{1}{c|}{10} & \multicolumn{1}{c|}{20} & \multicolumn{1}{c|}{40} & \multicolumn{1}{c|}{60} & \multicolumn{1}{c|}{80} & \multicolumn{1}{c|}{100} \\
\hline 0 & 6.6382 & 2.8214 & 2.3419 & 2.2769 & 2.2695 & 2.2675 & 2.2267 \\
1 & 10.8379 & 5.5257 & 4.6609 & 4.5163 & 4.4952 & 4.4885 & 4.4886 \\
2 & 11.8434 & 6.5080 & 6.1001 & 6.0042 & 5.9925 & 5.9892 & 5.9878 \\
5 & 12.8548 & 7.7374 & 6.8898 & 6.7802 & 6.7658 & 6.7616 & 6.7596 \\
10 & 13.8968 & 8.4799 & 7.6195 & 7.5014 & 7.4856 & 7.4808 & 7.4788 \\
Metal & 19.3594 & 13.3446 & 12.4627 & 12.3289 & 12.3108 & 12.3047 & 12.3026 \\
\hline
\end{tabular}


Table 3. Comparison of axial displacement, transverse deflection, normal stress and transverse shear stress in FG beams subjected to non-linear thermo-mechanical load $\left(L / h=10, T_{1}=10, T_{2}=10\right.$, $\left.q_{0}=100\right)$

\begin{tabular}{|cllrrrc|}
\hline$p$ & Theory & Model & $\bar{u}$ & $\bar{w}$ & $\bar{\sigma}_{x}$ & $\bar{\tau}_{x z}$ \\
\hline 0 & Present $\left(\varepsilon_{z} \neq 0\right)$ & FOSNDT & 0.5015 & 3.2265 & 9.8165 & 0.4768 \\
& Reddy $[79]$ & PSDT & 0.4991 & 3.2314 & 8.5113 & 0.4782 \\
\hline 1 & Present $\left(\varepsilon_{z} \neq 0\right)$ & FOSNDT & 0.7135 & 5.6475 & 12.5948 & 0.5086 \\
& Reddy $[79]$ & PSDT & 0.7065 & 5.7324 & 12.0543 & 0.5178 \\
\hline 2 & Present $\left(\varepsilon_{z} \neq 0\right)$ & FOSNDT & 0.8112 & 6.9469 & 13.7807 & 0.5472 \\
& Reddy [79] & PSDT & 0.8018 & 7.0821 & 13.6789 & 0.5489 \\
\hline 5 & Present $\left(\varepsilon_{z} \neq 0\right)$ & Present & 0.9353 & 8.1126 & 15.9134 & 0.5206 \\
& Reddy [79] & PSDT & 0.9220 & 8.3387 & 15.7291 & 0.5253 \\
\hline 10 & Present $\left(\varepsilon_{z} \neq 0\right)$ & FOSNDT & 1.0926 & 8.8763 & 18.5547 & 0.3987 \\
& Reddy [79] & PSDT & 1.0785 & 9.0378 & 18.3960 & 0.4203 \\
\hline$\infty$ & Present $\left(\varepsilon_{z} \neq 0\right)$ & FOSNDT & 2.1495 & 13.9323 & 23.4079 & 0.4815 \\
& Reddy [79] & PSDT & 2.1415 & 13.9289 & 23.0579 & 0.4769 \\
\hline
\end{tabular}

Table 4. Non-dimensional deflections $\bar{w}$ in FG beam subjected to non-linear thermo-mechanical load $\left(L / h=10, T_{1}=10, T_{2}=10\right)$

\begin{tabular}{|rrrrrrrr|}
\hline \multicolumn{7}{|c|}{ Aspect Ratio $(L / h)$} \\
\hline$p$ & \multicolumn{1}{c}{5} & 10 & \multicolumn{1}{c|}{20} & \multicolumn{1}{c|}{40} & \multicolumn{1}{c|}{60} & 80 & 100 \\
\hline 0 & 9.9887 & 9.9887 & 2.3923 & 2.2832 & 2.2773 & 2.2683 & 2.2671 \\
1 & 13.7517 & 5.6475 & 4.6382 & 4.5033 & 4.4883 & 4.4843 & 4.4828 \\
2 & 15.7436 & 6.9459 & 5.8188 & 5.6658 & 5.6484 & 5.6437 & 5.6419 \\
5 & 17.3946 & 8.1126 & 6.8901 & 6.7171 & 6.6973 & 6.6904 & 6.6881 \\
10 & 18.5259 & 8.8783 & 7.5945 & 7.4104 & 7.3878 & 7.3814 & 7.3788 \\
Metal & 25.7975 & 13.9323 & 12.3411 & 12.1148 & 12.0824 & 12.0797 & 12.0765 \\
\hline
\end{tabular}

Table 5. Non-dimensional deflections in FG beam for different models subjected to linear and non-linear thermo-mechanical load $\left(L / h=10, T_{1}=10, p=10, q_{0}=100\right)$

\begin{tabular}{|r|rrrr|rr|}
\hline \multirow{2}{*}{$L / h$} & \multicolumn{5}{|c|}{$T_{1}=10$} & \multicolumn{2}{c|}{$T_{1}=T_{2}=10$} \\
\cline { 2 - 7 } & \multicolumn{1}{|c}{ EBT } & \multicolumn{1}{c|}{ FSDT } & \multicolumn{1}{c|}{ Reddy } & \multicolumn{1}{c|}{ FOSNDT } & \multicolumn{1}{c|}{ Reddy } & FOSNDT \\
\hline 5 & 14.1128 & 13.8024 & 14.4925 & 14.3137 & 13.1670 & 13.4976 \\
10 & 8.3812 & 8.5689 & 8.9069 & 8.6311 & 7.9709 & 7.9878 \\
20 & 7.6648 & 7.7117 & 8.0971 & 7.8483 & 7.2328 & 7.2343 \\
40 & 7.5752 & 7.5869 & 7.9690 & 7.7264 & 7.1192 & 7.1191 \\
60 & 7.5662 & 7.5714 & 7.9514 & 7.7101 & 7.1042 & 7.1041 \\
80 & 7.5640 & 7.5669 & 7.9463 & 7.7052 & 7.0997 & 7.0996 \\
100 & 7.5632 & 7.5651 & 7.9441 & 7.7031 & 7.0979 & 7.0977 \\
\hline
\end{tabular}

axial displacement $\bar{u}$, transverse deflection $\bar{w}$, normal stress $\bar{\sigma}_{x}$ and transverse shear stress $\bar{\tau}_{x z}$ for FG beams subjected to non-linear thermo-mechanical loads. The observations are similar to the thermo-mechanical case except some marginal improvement in numerical values due to the inclusion of non-linear effect. Table 4 shows transverse deflection $\bar{w}$ for FG beam subjected to non-linear thermo-mechanical loads by varying aspect ratios. Tabulated results clearly show 
that transverse displacements are found larger in the case of non-linear thermo-mechanical loads compared to the linear load case. Table 5 shows the transverse displacements using different models when power law index $p=10$. It is observed that the present FOSNDT gives accurate results compared to other theories.

Fig. 4 shows the through thickness variation of axial displacement $\bar{u}$ for FG beam under linear and non-linear thermo-mechanical loadings, respectively. It is noticed that for the fully ceramic case $(p=0)$ the axial stresses are compressive in nature, whereas for fully metallic case $(p=\infty)$ it is tensile in nature. Fig. 5 shows the variation of transverse displacement $\bar{w}$ for FG beam under linear and non-linear thermo-mechanical load using different aspect ratios. In non-linear cases the displacements are slightly higher than in linear cases. Fig. 6 shows the variation of bending stress for the FG beam under linear and non-linear thermo-mechanical loads. The bending stress is zero at a neutral plane for ceramic and metal phases due to isotropic properties of the material and when $p=1,2,5,10$ there is a shift of the neutral plane due to continuous change in volume fraction across the thickness. It is important to observe that in the case of non-linear thermo-mechanical loads $\left(T_{1}=T_{2}=10\right)$ for $p=\infty$, the bending stress varies non-linearly. Fig. 7 compares the bending stress by CBT, TSDT and present FOSNDT to understand the accuracy of the present theory when compared with other theories taking into
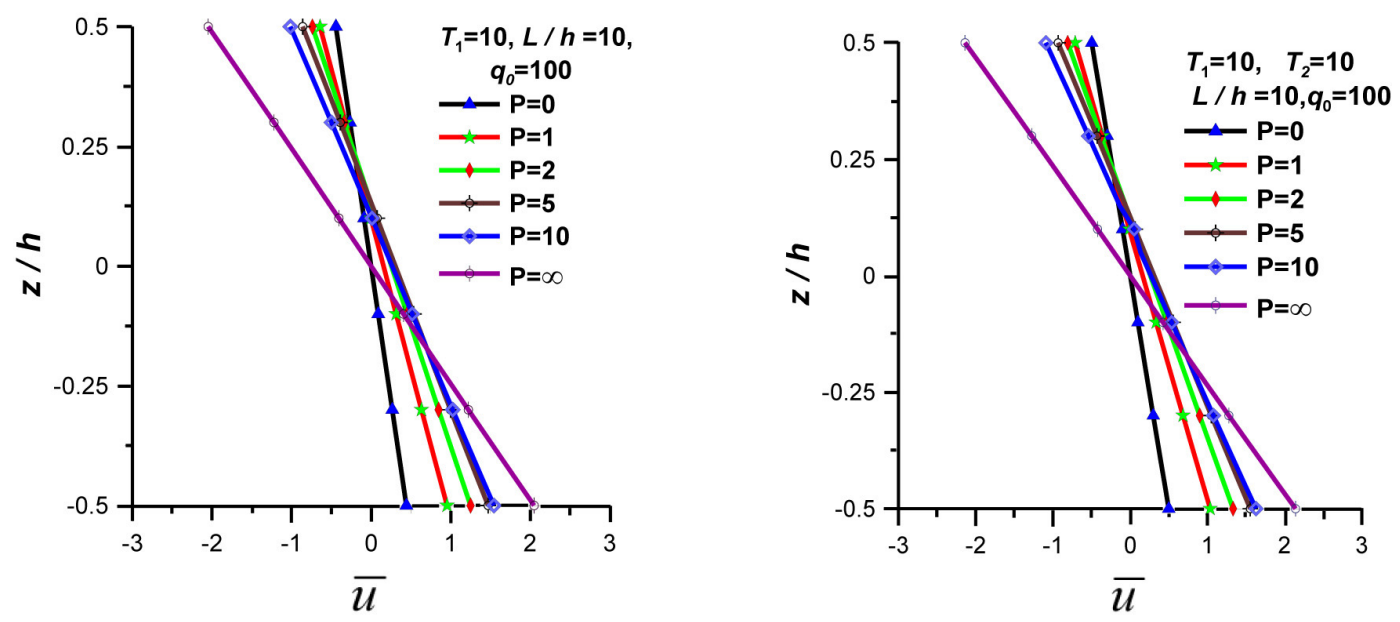

Fig. 4. Variations of non-dimensional axial displacement $\bar{u}$ across the thickness of FG beam subjected to linear (left) and non-linear (right) thermo-mechanical load
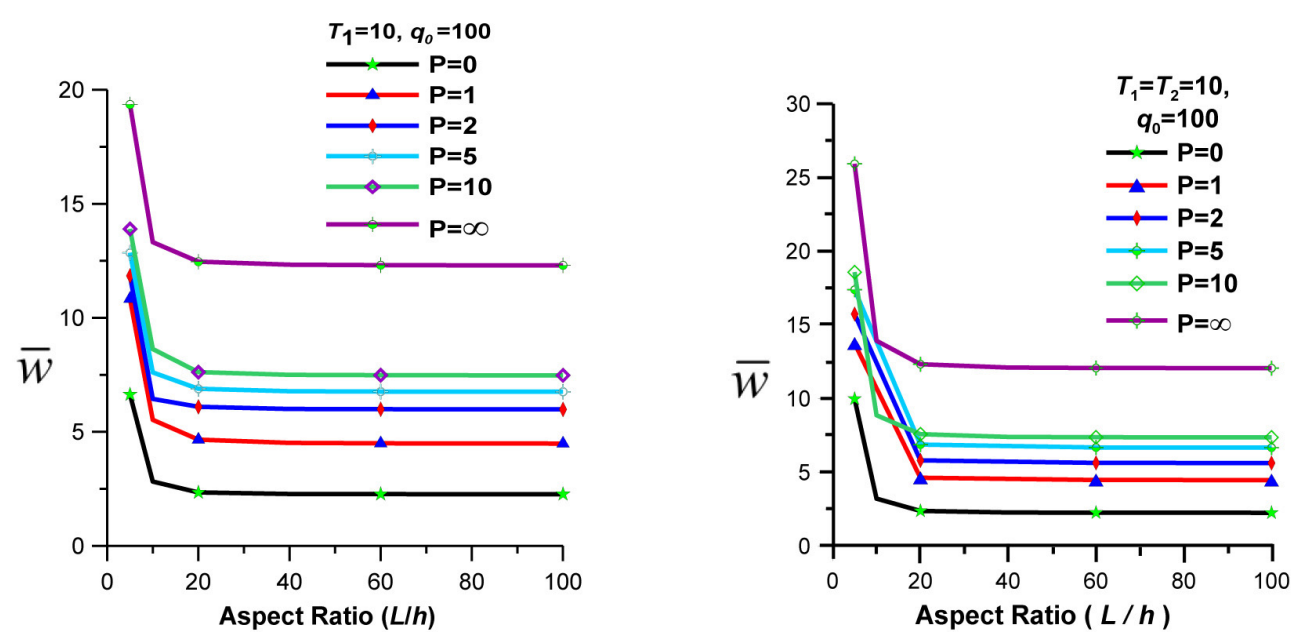

Fig. 5. Variations of non-dimensional transverse displacement across the thickness of FG beam subjected to linear (left) and non-linear (right) thermo-mechanical load for various aspect ratios 

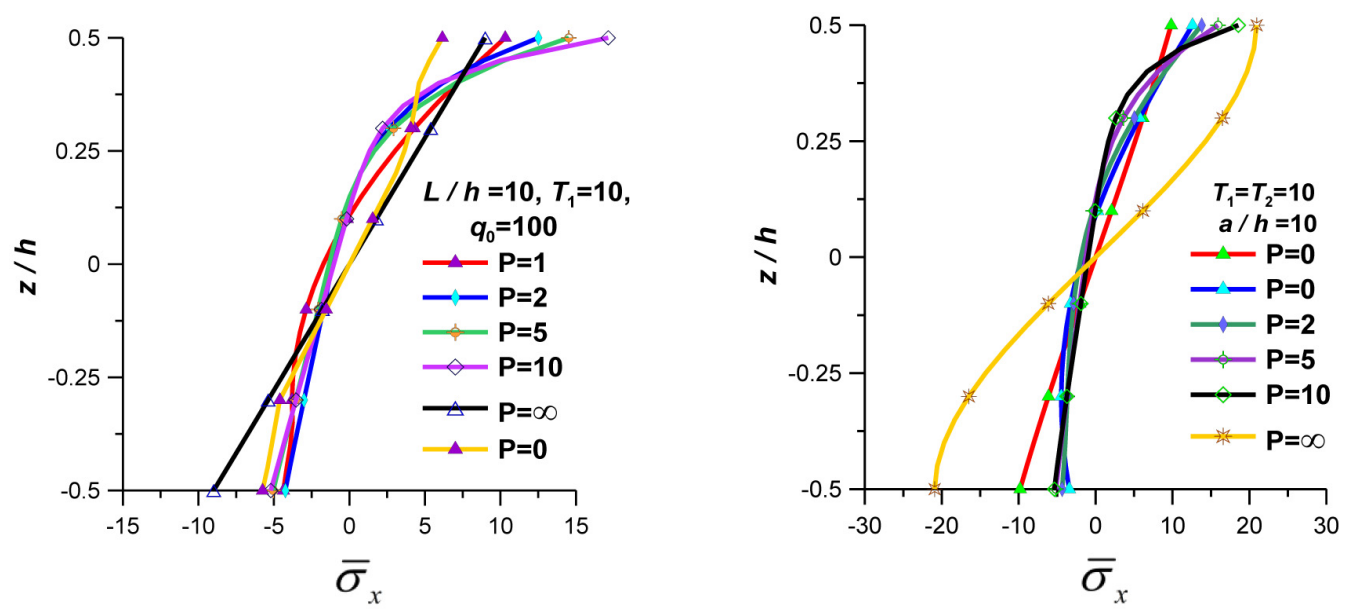

Fig. 6. Variations of non-dimensional bending stress across the thickness of FG beam subjected to linear (left) and non-linear (right) thermo-mechanical load
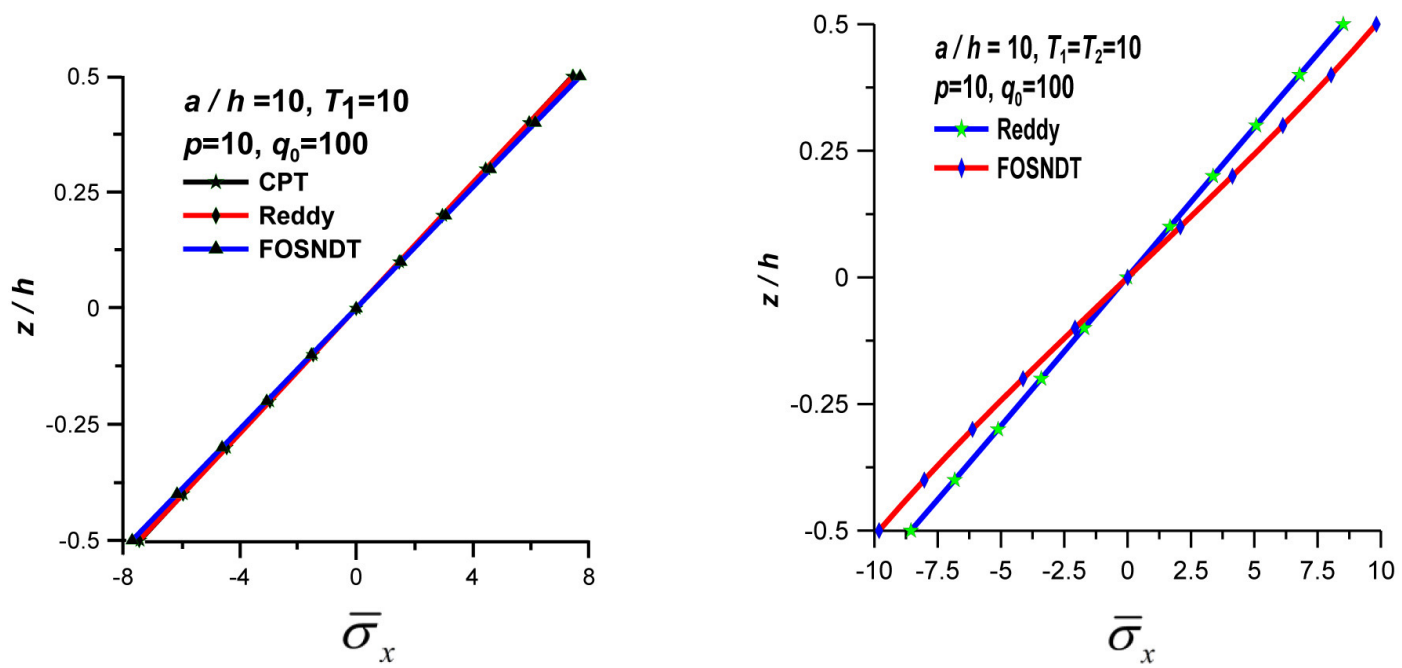

Fig. 7. Comparison of non-dimensional bending stress between CBT, Reddy and present theory for FG beam subjected to linear (left) and non-linear (right) thermo-mechanical load
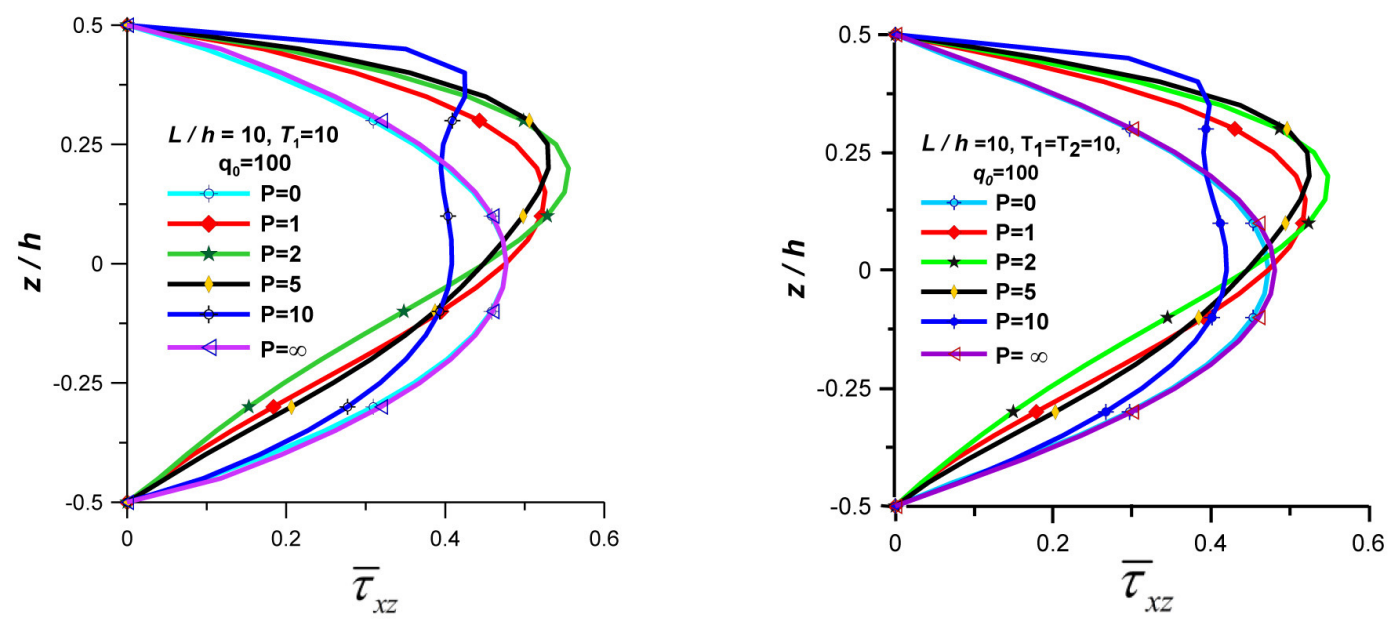

Fig. 8. Variations of non-dimensional transverse shear stress across the thickness of FG beam subjected to linear (left) and non-linear (right) thermo-mechanical load

account the effect of linear and non-linear thermo-mechanical loads. It is observed that bending stresses are higher in the case of non-linear loading case compared to the linear loading case. Fig. 8 shows the distribution of transverse shear stress through the thickness of FG beam. When 
$p=1,2,5$ and 10 due to the variation of volume fractions, the maximum values of transverse shear stress are found shifted above the neutral surface. For ceramic and metal phases, the transverse shear stress is a maximum at a neutral surface because of isotropic properties of materials.

\subsection{Numerical results for the hygro-thermo-mechanical load and their discussion}

Table 6 shows the comparison of displacements and stresses for FG beams subjected to linear hygro thermo-mechanical load. The comparison of thermo-mechanical and hygro-thermomechanical loading cases shows that the variations in stresses and displacements are similar in both cases, but due to the inclusion of moisture load the numerical values are greater for the hygo-thermo-mechanical case. It shows that the moisture load cannot be ignored to know the accurate behaviour of the FG beams. Table 7 shows the maximum non-dimensional transverse deflection under linear hygro-thermo-mechanical loads considering different aspect ratios. As the aspect ratio increases, the deflection decreases. Table 8 shows the comparison of maximum non-dimensional axial displacement $\bar{u}$, transverse deflection $\bar{w}$, normal stress $\bar{\sigma}_{x}$ and transverse shear stress $\bar{\tau}_{x z}$ for FG beams subjected to non-linear hygro-thermo-mechanical loads. In case of axial displacement $\bar{u}$, it is noticed that the axial displacement values obtained by present

Table 6. Comparison of axial displacement, transverse deflection, normal stress and transverse shear stress in FG beams subjected to linear hygro-thermo-mechanical load $\left(L / h=10, T_{1}=10, C_{1}=100\right.$, $\left.q_{0}=100\right)$

\begin{tabular}{|cllcrrc|}
\hline$p$ & Theory & Model & $\bar{u}$ & $\bar{w}$ & $\bar{\sigma}_{x}$ & $\bar{\tau}_{x z}$ \\
\hline 0 & Present $\left(\varepsilon_{z} \neq 0\right)$ & FOSNDT & 0.4490 & 2.8591 & 7.7742 & 0.4846 \\
& Reddy $[79]$ & PSDT & 0.4445 & 2.8186 & 7.4529 & 0.4773 \\
\hline \multirow{2}{*}{1} & Present $\left(\varepsilon_{z} \neq 0\right)$ & FOSNDT & 0.9921 & 5.6349 & 10.8664 & 0.5199 \\
& Reddy [79] & PSDT & 1.0080 & 5.2915 & 11.0799 & 0.5186 \\
\hline 2 & Present $\left(\varepsilon_{z} \neq 0\right)$ & FOSNDT & 1.3028 & 6.9560 & 12.9832 & 0.5532 \\
& Reddy [79] & PSDT & 1.3170 & 6.8260 & 12.7559 & 0.5482 \\
\hline 5 & Present $\left(\varepsilon_{z} \neq 0\right)$ & Present & 1.5759 & 8.2455 & 14.9450 & 0.5266 \\
& Reddy $[79]$ & PSDT & 1.5827 & 8.1312 & 14.8367 & 0.5209 \\
\hline 10 & Present $\left(\varepsilon_{z} \neq 0\right)$ & FOSNDT & 1.6072 & 8.7925 & 17.6673 & 0.4138 \\
& Reddy [79] & PSDT & 1.6307 & 8.6415 & 17.5639 & 0.4119 \\
\hline$\infty$ & Present $\left(\varepsilon_{z} \neq 0\right)$ & FOSNDT & 2.1241 & 13.5488 & 21.7548 & 0.4769 \\
& Reddy [79] & PSDT & 2.1338 & 13.3789 & 21.5123 & 0.4724 \\
\hline
\end{tabular}

Table 7. Non-dimensional deflections $\bar{w}$ in FG beam subjected to linear hygro-thermo-mechanical load $\left(T_{1}=10, C_{1}=100, q_{0}=100\right)$

\begin{tabular}{|rrrrrrrr|}
\hline \multicolumn{7}{|c|}{ Aspect Ratio $(L / h)$} \\
\hline$p$ & \multicolumn{1}{c}{5} & \multicolumn{1}{c|}{10} & \multicolumn{1}{c|}{20} & \multicolumn{1}{c|}{40} & \multicolumn{1}{c|}{60} & 80 & \multicolumn{1}{c|}{100} \\
\hline 0 & 6.9571 & 2.8591 & 2.3405 & 2.2774 & 2.2696 & 2.2676 & 2.2667 \\
1 & 12.7817 & 5.6349 & 4.6743 & 4.5179 & 4.4956 & 4.4887 & 4.4857 \\
2 & 12.8520 & 6.9560 & 6.0028 & 5.8923 & 5.8791 & 5.8754 & 5.8739 \\
5 & 14.0014 & 8.2455 & 6.9072 & 6.7824 & 6.7685 & 6.7618 & 6.7599 \\
10 & 15.6707 & 8.7925 & 7.8686 & 7.7290 & 7.7055 & 7.7039 & 7.7031 \\
Metal & 21.3034 & 13.5488 & 12.4919 & 12.3325 & 12.3119 & 12.3058 & 12.3033 \\
\hline
\end{tabular}


Table 8. Comparison of axial displacement, transverse deflection, normal stress and transverse shear stress in FG beams subjected to non-linear hygro-thermo-mechanical load $\left(L / h=10, T_{1}=T_{2}=10\right.$, $C_{1}=C_{2}=10, q_{0}=100$ )

\begin{tabular}{|cllcrrc|}
\hline$p$ & Theory & Model & $\bar{u}$ & $\bar{w}$ & $\bar{\sigma}_{x}$ & $\bar{\tau}_{x z}$ \\
\hline 0 & Present $\left(\varepsilon_{z} \neq 0\right)$ & FOSNDT & 0.5129 & 3.2641 & 9.8935 & 0.4810 \\
& Reddy $[79]$ & PSDT & 0.5065 & 3.2314 & 8.5563 & 0.4763 \\
\hline \multirow{2}{*}{1} & Present $\left(\varepsilon_{z} \neq 0\right)$ & FOSNDT & 1.0944 & 6.2265 & 13.6307 & 0.5194 \\
& Reddy $[79]$ & PSDT & 1.1145 & 5.8904 & 12.3530 & 0.5170 \\
\hline 2 & Present $\left(\varepsilon_{z} \neq 0\right)$ & FOSNDT & 1.4157 & 7.3720 & 15.6969 & 0.5481 \\
& Reddy [79] & PSDT & 1.4370 & 7.3153 & 14.0983 & 0.5462 \\
\hline 5 & Present $\left(\varepsilon_{z} \neq 0\right)$ & Present & 1.7122 & 8.9595 & 17.8682 & 0.5465 \\
& Reddy $[79]$ & PSDT & 1.7272 & 8.8702 & 16.2201 & 0.5195 \\
\hline 10 & Present $\left(\varepsilon_{z} \neq 0\right)$ & FOSNDT & 1.7761 & 9.4502 & 20.8281 & 0.4266 \\
& Reddy $[79]$ & PSDT & 1.7820 & 9.3278 & 19.0277 & 0.3980 \\
\hline$\infty$ & Present $\left(\varepsilon_{z} \neq 0\right)$ & FOSNDT & 2.2524 & 14.5497 & 25.0805 & 0.4811 \\
& Reddy $[79]$ & PSDT & 2.2467 & 14.5146 & 24.1430 & 0.4769 \\
\hline
\end{tabular}

Table 9. Non dimensional deflections $\bar{w}$ in FG beam subjected to non-linear hygro-thermo-mechanical load $\left(T_{1}=T_{2}=10, C_{1}=C_{2}=10, q_{0}=100\right)$

\begin{tabular}{|ccrrrrrr|}
\hline \multicolumn{7}{c|}{ Aspect Ratio $(L / h)$} \\
\hline$p$ & \multicolumn{1}{c}{5} & \multicolumn{1}{c|}{10} & \multicolumn{1}{c|}{20} & \multicolumn{1}{c|}{40} & \multicolumn{1}{c|}{60} & 80 & \multicolumn{1}{c|}{100} \\
\hline 0 & 10.3076 & 3.2641 & 2.3969 & 2.2837 & 2.2715 & 2.2684 & 2.2672 \\
1 & 18.0711 & 6.2265 & 4.7460 & 4.5268 & 4.4983 & 4.4898 & 4.4862 \\
2 & 18.2340 & 7.3720 & 6.1405 & 5.9594 & 5.9337 & 5.9301 & 5.9286 \\
5 & 19.0992 & 8.9595 & 6.9840 & 6.7920 & 6.7630 & 6.7618 & 6.7605 \\
10 & 20.5869 & 9.4502 & 7.7193 & 7.5138 & 7.4824 & 7.4811 & 7.4796 \\
Metal & 27.7811 & 14.5497 & 12.5881 & 12.3446 & 12.3073 & 12.3055 & 12.3041 \\
\hline
\end{tabular}

Table 10. Non dimensional deflections $\bar{w}$ in FG beam for different models subjected to linear and non-linear hygro-thermo-mechanical load $\left(p=10, q_{0}=100\right)$

\begin{tabular}{|r|rrrr|rc|}
\hline \multirow{2}{*}{$L / h$} & \multicolumn{5}{|c|}{$T_{1}=10, C_{1}=100$} & \multicolumn{2}{c|}{$T_{1}=T_{2}=10, C_{1}=C_{2}=100$} \\
\cline { 2 - 7 } & \multicolumn{1}{|c}{ EBT } & \multicolumn{1}{c|}{ FSDT } & \multicolumn{1}{c|}{ Reddy } & FOSNDT & \multicolumn{1}{c|}{ Reddy } & \multicolumn{1}{c|}{ FOSNDT } \\
\hline 5 & 15.3849 & 16.1357 & 15.0678 & 15.6727 & 20.2472 & 21.2043 \\
10 & 8.5402 & 8.7279 & 8.6415 & 8.7925 & 9.3278 & 9.4502 \\
20 & 7.6886 & 7.7316 & 7.7314 & 7.8686 & 7.8188 & 7.9509 \\
40 & 7.5777 & 7.5854 & 7.5920 & 7.7290 & 7.6030 & 7.7393 \\
60 & 7.5669 & 7.5721 & 7.5737 & 7.7109 & 7.5769 & 7.7139 \\
80 & 7.5643 & 7.5673 & 7.5682 & 7.7055 & 7.5696 & 7.7068 \\
100 & 7.5634 & 7.5653 & 7.5659 & 7.7033 & 7.5666 & 7.7040 \\
\hline
\end{tabular}

FOSNDT are higher for ceramic and metal phases, whereas for other power index values it is less than PSDT. The transverse deflection $\bar{w}$, normal stresses $\bar{\sigma}_{x}$ and transverse shear stresses $\bar{\tau}_{x z}$ obtained by present FOSNDT are greater than PSDT for all the values of power law index. Other variations in displacements and stresses are observed similar to thermo mechanical loading cases except some marginal improvement in the results due to the inclusion of hygro load. Table 9 shows maximum deflection for FG beam subjected to non-linear hygro-thermo- 
mechanical loading for various aspect ratio and power law index. As the aspect ratio increases, the non-dimensional deflection decreases and as the power law index increases, the deflection increases due to increase in flexibility of the FG beams. The displacements are noticeably greater in non-linear hygro-thermo-mechanical load than in the linear load case. Table 10 shows the results of transverse displacements for different models for power law index $p=10$, it is observed that all the displacement values are having a good agreement with other theories.

Fig. 9 shows the axial displacements $\bar{u}$ across the FG beams subjected to linear and non-linear hygro-thermo-mechanical loadings, respectively. In the non-linear case, the axial displacements are larger. Fig. 10 shows the variation of transverse displacements $\bar{w}$ for FG beams subjected to linear and non-linear hygro-thermo-mechanical loads. Due to low stiffness when $p=\infty$, the displacements are noticeably larger. Fig. 11 shows the bending stress variation for FG beams subjected to linear and non-linear hygro-thermo-mechanical loads. The variations are exactly similar to the hygro-thermo-mechanical loading case. It is important to notice that in non-linear hygro-thermo-mechanical loads when $p=\infty$, the bending stress varies non-linearly. Fig. 12 shows the distribution of transverse shear stress through the thickness of the FG beam for thermo-mechanical and hygro-thermo-mechanical loading cases. For ceramic and metal phases,
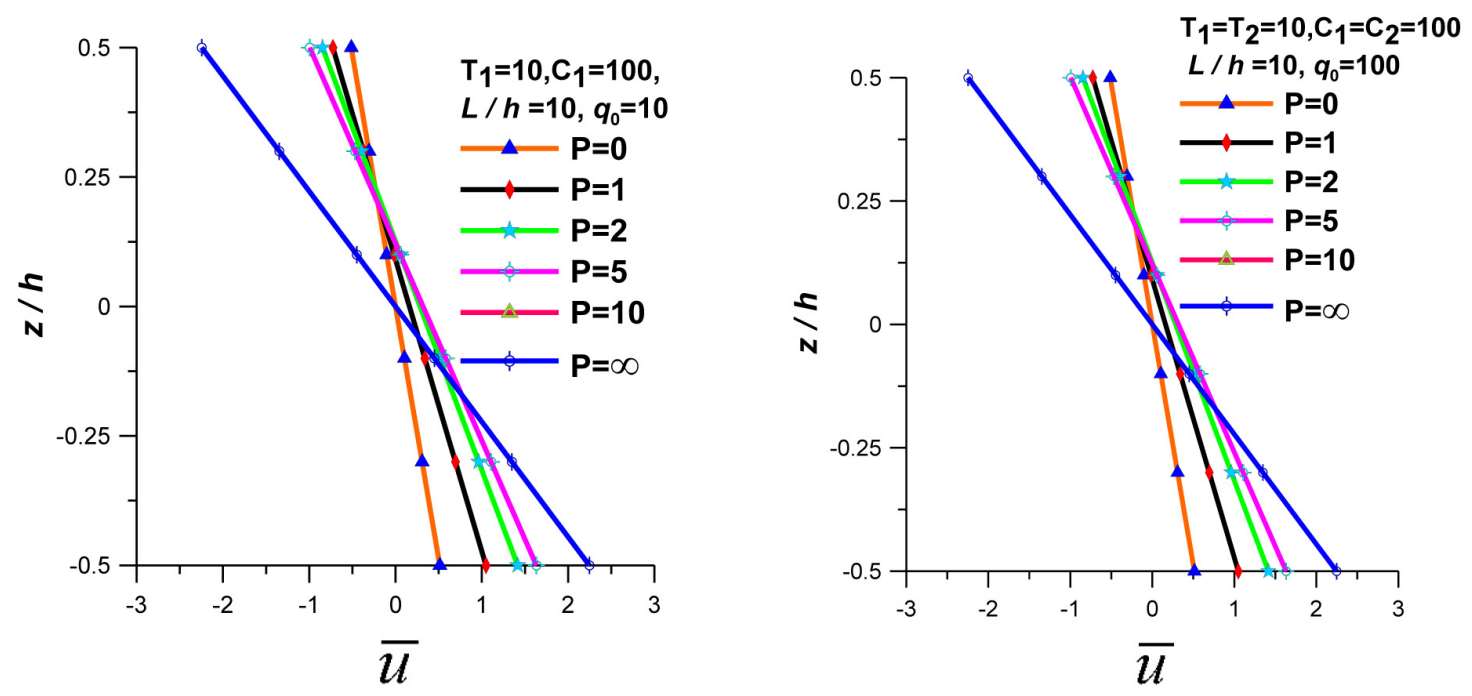

Fig. 9. Variations of axial displacement $\bar{u}$ across the thickness of simply supported FG beams subjected to linear (left) and non-linear (right) hygro-thermo-mechanical load
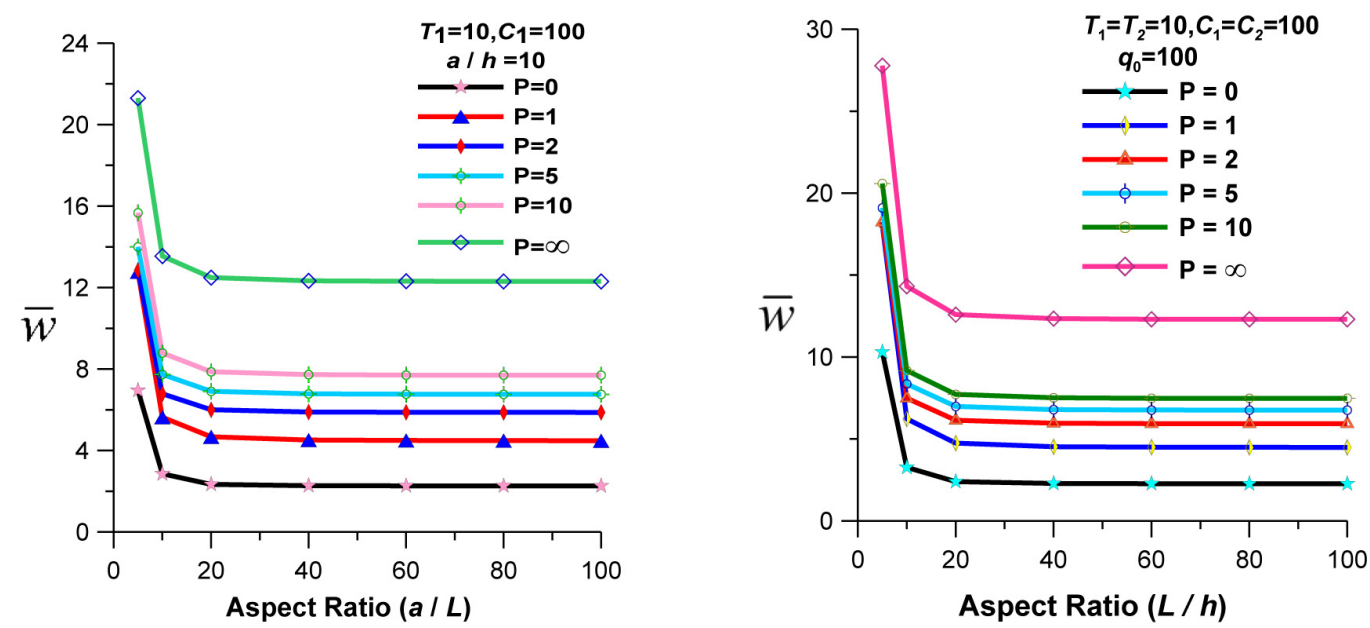

Fig. 10. Variations of displacement $\bar{w}$ across the thickness of FG beams subjected to linear (left) and non-linear (right) hygro-thermo-mechanical load for various aspect ratios 

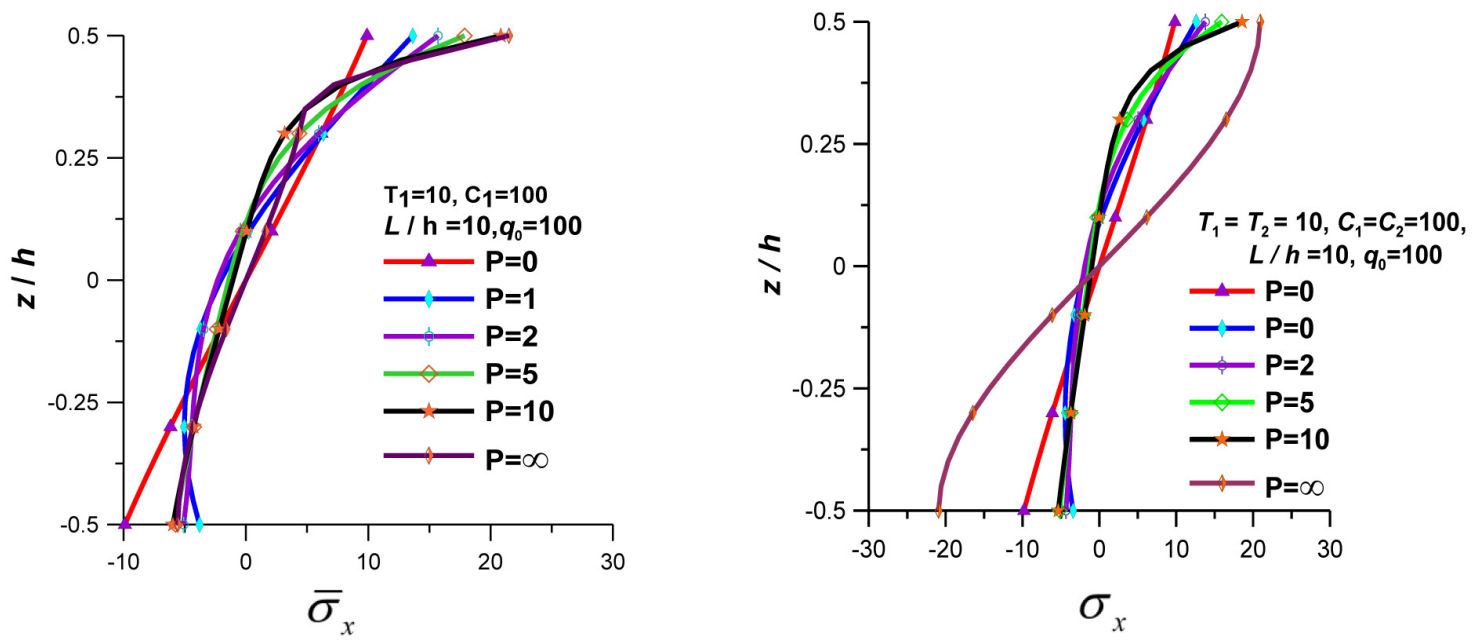

Fig. 11. Variations of bending stress $\bar{\sigma}_{\mathbf{x}}$ across the thickness FG beams subjected to linear (left) and non-linear (right) hygro-thermo-mechanical loads
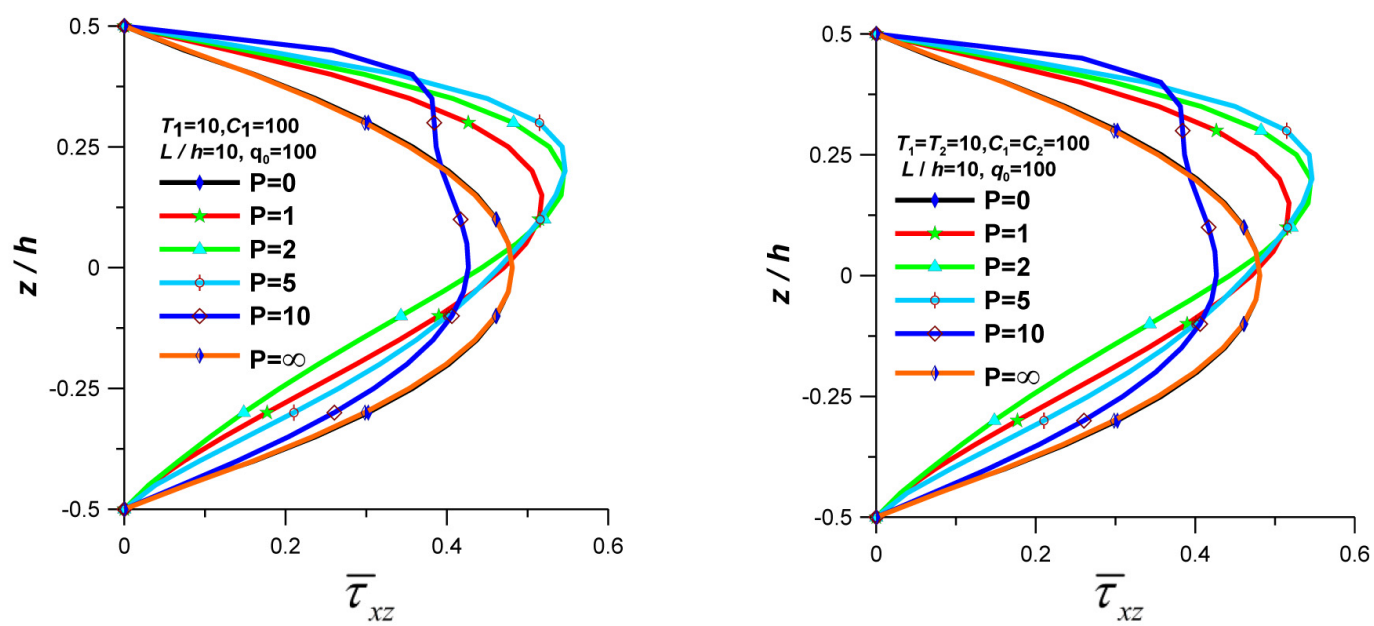

Fig. 12. Variations of transverse shear stress $\bar{\tau}_{x z}$ across the thickness of FG beams subjected to linear (left) and non-linear (right) hygro-thermo-mechanical loads

the transverse shear stress has a maximum at the neutral surface because of isotropic material properties. But when $p=1,2,5$ and 10 due to the variation of volume fractions, the maximum values of transverse shear stress are found shifted from the neutral surface in metal phase. Other variations are noticeable, similar to the thermo-mechanical loading case.

\section{Conclusions}

The present study includes the effect of non-linear hygro-thermo-mechanical loading and thickness stretching to predict the accurate bending response of the FG beams using a fifthorder shear and normal deformation theory (FOSNDT). FOSNDT includes the fourth order variation of transverse displacement and fifth-order variation of axial displacements. This theory does not need the shear correction factor to satisfy the traction free boundary conditions of the FG beam. Using the principle of virtual displacement, the variationally consistent governing equations and associated boundary conditions are obtained. Using the Navier's solution technique, the analytical solution is obtained for simply supported FG beam. Several important observations from the present study can be formulated: 
1. Displacements are increasing with an increase in the power law index due to the increase in the flexibility with an increase in the power law index.

2. The analysis of obtained results shows that the displacements and stresses are marginally higher in non-linear hygro-thermo mechanical loading cases than in cases with linear loading.

3. It is important to notice that in the case of non-linear hygro-thermo-mechanical loads when $p=\infty$, the bending stress varies non-linearly.

4. The present theory is very simple to predict the accurate bending response of FG beams in the presence of both thermal and hygro loads.

5. The numerical results by the present FOSNDT are in excellent agreement with other theories.

\section{Important recommendations}

From the numerical results and observations, authors recommendations are as follows:

1. For hygro-thermo-mechanical analysis Carrera recommended that thickness cocordinate must be expanded at least up to the fifth order to get the accurate results for FG beams and plates.

2. Since the temperature and moisture effects are changing through the thickness, it is recommended to consider the thickness stretching effect to get the accurate results when FG beams are subjected to hygro-thermo-mechanical load.

3. As per the authors' knowledge, the results obtained by the present investigation with the hygro-thermo-mechanical loading will be the benchmark for future researchers and scientists in the area which is addressed for FG beam first time.

Scope for the future work

It will be very interesting to address the effect of hygro-thermo-mechanical load on FG beam considering the following points:

1. Dynamic and buckling response of FG beams using FOSNDT under non-linear hygrothermo mechanical loadings considering the thickness stretching.

2. To analyze the FG beams for other boundary conditions using FOSNDT considering the thickness stretching and non-linear hygro-thermo mechanical loading.

3. To study the bending the behaviour of the curved beams and shells using FOSNDT with the thermal and moisture effect.

\section{References}

[1] Abdelhak, Z., Hadji, L., Daouadji, T. H., Adda Bedia, E. A., Thermal buckling response of functionally graded sandwich plates with clamped boundary conditions, Smart Structures and Systems 18 (2) (2016) 267-291. https://doi.org/10.12989/sss.2016.18.2.267

[2] Abdelhak, Z., Hadji, L., Khelifa, Z., Daouadji, T.H., Adda Bedia, E. A., Analysis of buckling response of functionally graded sandwich plates using a refined shear deformation theory, Wind and Structures 22 (3) (2016) 291-305. https://doi.org/10.12989/was.2016.22.3.291 
[3] Aboudi, J., Arnold, S. M., Pindera, M. J., Response of functionally graded composites to thermal gradients, Composites Engineering 4 (1994) 1-18. https://doi.org/10.1016/0961-9526(94)90003-5

[4] Adim, B., Daouadji, T.H., Effects of thickness stretching in FGM plates using a quasi-3D higher order shear deformation theory, Advances in Materials Research 5 (4) (2016) 223-244. https://doi.org/10.12989/amr.2016.5.4.223

[5] Adim, B., Daouadji, T.H., Abbes, B., Buckling analysis of anti-symmetric cross-ply laminated composite plates under different boundary conditions, International Applied Mechanics 52 (6) (2016) 126-141. https://doi.org/10.1007/s10778-016-0787-x

[6] Adim, B., Daouadji, T.H., Abdrezak, R., Amine, B.M., Zidour, M., Abbes, B., Mechanical buckling analysis of hybrid laminated composite plates under different boundary conditions, Structural Engineering and Mechanics 66 (6) (2018) 761-769.

[7] Adim, B., Daouadji, T.H., Abdrezak, R., Tounsi, A., An efficient and simple higher order of shear deformation theory for static and free vibration of laminated composite plates, International International Journal of Composite Materials and Matrices 2 (1) (2016) 23-42.

[8] Adim, B., Daouadji, T.H., Rabia, B., Hadji, L., An efficient and simple higher order shear deformation theory for bending analysis of composite plates under various boundary conditions, Earthquakes and Structures 11 (1) (2016) 63-82. https://doi.org/10.12989/eas.2016.11.1.063

[9] Arbind, A., Reddy, J. N., Srinivasa, A. R., Modified couple stress-based third-order theory for nonlinear analysis of functionally graded beams, Latin American Journal of Solids and Structures 11 (2014) 459-487. https://doi.org/10.1590/S1679-78252014000300006

[10] Benatta, M. A., Mechab, I., Tounsi, A., Bedia, E. A. A., Static analysis of functionally graded short beams including warping and shear deformation effects, Computational Materials Science 44 (2008) 765-773. https://doi.org/10.1016/j.commatsci.2008.05.020

[11] Benferhat, R., Daouadji, T. H., Abderezak, R., Effect of distribution shape of the porosity on the interfacial stresses of the FGM beam strengthened with FRP plate, Earthquakes and Structures 16 (5) (2019) 601-609.

[12] Benferhat, R., Daouadji, T.H., Abderezak, R., Effect of porosity in interfacial stress analysis of perfect FGM beams reinforced with a porous functionally graded materials plate, Structural Engineering and Mechanics 72 (3) (2019) 293-304.

[13] Benferhat, R., Daouadji, T. H., Adim, B., A novel higher order shear deformation theory based on the neutral surface concept of FGM plate under transverse load, Advances in Materials Research 5 (2) (2016) 107-120. https://doi.org/10.12989/amr.2016.5.2.107

[14] Benferhat, R., Daouadji, T.H., Hadji, L., Said, M. M., Static analysis of the FGM plate with porosities, Steel and Composite Structures 21 (1) (2016) 123-136. https://doi.org/10.12989/scs.2016.21.1.123

[15] Benferhat, R., Daouadji, T. H., Mansour, M. S., Free vibration analysis of FG plates resting on the elastic foundation and based on the neutral surface concept using higher order shear deformation theory, Comptes Rendus Mecanique 344 (9) (2016) 631-641.

https://doi.org/10.1016/j.crme.2016.03.002

[16] Benferhat, R., Daouadji, T.H., Mansour, M. S., Hadji, L., Effect of porosity on the bending and free vibration response of functionally graded plates resting on Winkler-Pasternak foundations, Eartquakes and Structures 10 (5) (2016) 1429-1449. https://doi.org/10.12989/eas.2016.10.6.1429

[17] Benferhat, R., Daouadji, T.H., Rabahi, A., Effect of distribution shape of the porosity on the interfacial stresses of the FGM beam strengthened with FRP plate, Earthquakes and Structures 16 (5) (2019) 601-609.

[18] Benhenni, M.A., Adim, B., Daouadji, T.H., Abbes, B., Abbes, F., Li, Y., Bouzidane, A., A comparison of closed form and finite element solutions for the free vibration of hybrid cross ply laminated plates, Mechanics of Composite Materials 55 (2) (2019) 181-194. https://doi.org/10.1007/s11029-019-09803-2 
[19] Benhenni, M. A., Daouadji, T. H., Abbes, B., Abbes, F., Li, Y., Adim, B., Numerical analysis for free vibration of hybrid laminated composite plates for different boundary conditions, Structural Engineering and Mechanics 70 (5) (2019) 535-549.

[20] Benhenni, M. A., Daouadji, T. H., Abbes, B., Adim, B., Li, Y., Abbes, F., Dynamic analysis for antisymmetric cross-ply and angle-ply laminates for simply supported thick hybrid rectangular plates, Advances in Materials Research 7 (2) (2018) 119-136.

[21] Benhenni, M. A., Daouadji, T. H., Abbes, B., Li, Y. M., Analytical and numerical results for free vibration of laminated composites plates, World Academy of Science, Engineering and Technology, International Journal of Chemical and Molecular Engineering 12 (6) (2018) 300-304.

[22] Bensattalah, T., Zidour, M., Daouadji, T.H., A new nonlocal beam model for free vibration analysis of chiral single-walled carbon nanotubes, Composite Materials and Engineering 1 (1) (2019) 21-31.

[23] Bensattalah, T., Zidour, M., Daouadji, T. H., Analytical analysis for the forced vibration of CNT surrounding elastic medium including thermal effect using nonlocal Euler-Bernoulli theory, Advances in Materials Research 7 (3) (2018) 163-174.

[24] Bensattalah, T., Zidour, M., Daouadji, T. H., Bouakaz, K., Theoretical analysis of chirality and scale effects on critical buckling load of zigzag triple walled carbon nanotubes under axial compression embedded in polymeric matrix, Structural Engineering and Mechanics 70 (3) (2019) 269-277.

[25] Birman, V., Byrd, L. W., Modeling and analysis of functionally graded materials and structures, Applied Mechanics Reviews 60 (2007) 195-216. https://doi.org/10.1115/1.2777164

[26] Bourada, M., Kaci, A., Houari, M. S. A., Tounsi, A., A new simple shear and normal deformations theory for functionally graded beams, Steel Composite Structures 18 (2015) 409-423. https://doi.org/10.12989/scs.2015.18.2.409

[27] Chakraborty, A., Gopalakrishnan, V., Reddy, J. N., A new beam finite element for the analysis of functionally graded materials, International Journal of Mechanics Sciences 45 (2003) 519-539. https://doi.org/10.1016/S0020-7403(03)00058-4

[28] Chedad, A., Daouadji, T. H., Abdrezak, R., Adim, B., Abbes, B., Rabia, B., Abbes, F., A highorder closed-form solution for interfacial stresses in externally sandwich FGM plated RC beams, Advances in Materials Research 6 (4) (2017) 317-328.

[29] Chergui, S., Daouadji, T. H., Hamrat, M., Boulekbache, B., Bougara, A., Abbes, B., Amziane, S., Interfacial stresses in damaged RC beams strengthened by externally bonded prestressed GFRP laminate plate: Analytical and numerical study, Advances in Materials Research 8 (3) (2019) $197-217$.

[30] Chin, H. K., Chen, J. K., Thermal stress analysis of functionally graded composites with temperaturedependent material properties, Journal of Mechanics of Materials and Structure 2 (4) (2007) 633-654. https://doi.org/10.2140/jomms.2007.2.633

[31] Chu, P., Li, X.F., Wu, J.X., Lee, K. Y., Two-dimensional elasticity solution of elastic strips and beams made of functionally graded materials under tension and bending, Acta Mechanica 226 (2015) 2235-2253. https://doi.org/10.1007/s00707-014-1294-y

[32] Daouadji, T.H., Adim, B., Benferhat, R., Bending analysis of an imperfect FGM plates under hygro-thermo-mechanical loading with analytical validation, Advances in Materials Research 5 (1) (2016) 35-53. https://doi.org/10.12989/amr.2016.5.1.035

[33] Daouadji, T. H., Adim, B., Mechanical behaviour of FGM sandwich plates using a quasi-3D higher order shear and normal deformation theory, Structural Engineering and Mechanics 61 (1) (2017) 49-63. https://doi.org/10.12989/sem.2017.61.1.049

[34] Daouadji, T. H., Adim, B., Theoretical analysis of composite beams under uniformly distributed load, Advances in Materials Research 5 (1) (2016) 1-9. https://doi.org/10.12989/amr.2016.5.1.001

[35] Daouadji, T. H., Boussad, A. S., Abderezak, R., Benferhat, R., Fazilay, A., Belkacem, A., Flexural behaviour of steel beams reinforced by carbon fibre reinforced polymer: Experimental and numerical study, Structural Engineering and Mechanics 72 (4) (2019) 409-419. 
[36] Daouadji, T.H., Henni, A.H., Tounsi, A., Bedia, E.A.A., Elasticity solution of a cantilever functionally graded beam, Applied Composite Materials 20 (2013) 1-15. https://doi.org/10.1007/s10443-011-9243-6

[37] Daouadji, T.H., Rabia, B., Adim, B., Bending analysis of an imperfect advanced composite plates resting on the elastic foundations, Coupled Systems Mechanics 5 (3) (2016) 269-283. https://doi.org/10.12989/csm.2016.5.3.269

[38] Ding, H.J., Huang, D. J., Chen, W. Q., Elasticity solutions for plane anisotropic functionally graded beams, International Journal of Solids and Structures 44 (1) (2007) 176-196. https://doi.org/10.1016/j.ijsolstr.2006.04.026

[39] Esfahani, S.E., Kiani, Y., Eslami, M. R., Non-linear thermal stability analysis of temperature dependent FGM beams supported on non-linear hardening elastic foundations, International Journal of Mechanical Sciences 69 (2013) 10-20. https://doi.org/10.1016/j.ijmecsci.2013.01.007

[40] Euler, L. Methodus inveniendi lineas curvas maximi minimive proprietate gaudentes. Appendix: De curvis elastics, Lausanne and Geneva, (1744) from opera omnia, Vol I edited by A. Speiser, E. Trost and Ch. Blanc. Zurich: Orell Fussli, (1952) 231.

[41] Filippi, M., Carrera, E., Zenkour, A. M., Static analyses of FGM beams by various theories and finite elements, Composites Part-B 72 (2015) 1-9. https://doi.org/10.1016/j.compositesb.2014.12.004

[42] Frikha, A., Hajlaoui, A., Wali, M., Dammak, F., A new higher order $\mathrm{C}^{0}$ mixed beam element for FGM beams analysis, Composites Part-B 106 (2016) 181-189. https://doi.org/10.1016/j.compositesb.2016.09.024

[43] Ghumare, S. M., Sayyad, A. S., A new fifth-order shear and normal deformation theory for static bending and elastic buckling of P-FGM beams, Latin American Journal of Solids and Structures 14 (2017) 1893-1911. https://doi.org/10.1590/1679-78253972

[44] Ghumare, S. M., Sayyad, A. S., A new quasi-3D model for functionally graded plates, Journal of Applied and Computational Mechanics 5 (2) (2019) 367-380.

[45] Ghumare, S. M., Sayyad, A. S., Analysis of functionally graded plates resting on elastic foundation and subjected to non-linear hygro-thermo-mechanical loadings. JMST Advances 1 (2019) 233-248. https://doi.org/10.1007/s42791-019-00024-1

[46] Ghumare, S. M., Sayyad, A. S., Non-linear hygro-thermo-mechanical analysis of functionally graded plates using a fifth order plate theory, Arabian Journal for Science and Engineering 44 (10) (2019) 8727-8745. https://doi.org/10.1007/s13369-019-03894-8

[47] Giunta, G., Belouettar, S., Carrera, E., Analysis of FGM beams by means of classical and advanced theories, Mechanics of Advanced Materials and Structures 17 (8) (2010) 622-635. https://doi.org/10.1080/15376494.2010.518930

[48] Giunta, G., Crisafulli, D., Belouettar, S., Carrera, E., A thermo-mechanical analysis of functionally graded beams via hierarchical modelling, Composite Structures 95 (2013) 676-690. https://doi.org/10.1016/j.compstruct.2012.08.013

[49] Giunta, G., Crisafulli, D., Belouettar, S., Carrera, E., Hierarchical theories for the free vibration analysis of functionally graded beams, Composite Structures 94 (1) (2011) 68-74. https://doi.org/10.1016/j.compstruct.2011.07.016

[50] Hadj, B., Benferhat, R., Daouadji, T. H., Influence of the distribution shape of porosity on the bending FGM new plate model resting on elastic foundations, Structural Engineering and Mechanics 72 (1) (2019) 61-70.

[51] Hadji, L., Daouadji, T. H., Bedia, E. A., Dynamic behavior of FGM beam using a new first shear deformation theory, Earthquakes and Structures 10 (2) (2016) 451-461. https://doi.org/10.12989/eas.2016.10.2.451

[52] Hadji, L., Daouadji, T.H., Meziane, M.A. A., Tlidji, Y., Bedia, E.A.A., Analysis of functionally graded beam using a new first-order shear deformation theory, Structural Engineering and Mechanics 57 (2016) 315-325. https://doi.org/10.12989/sem.2016.57.2.315 
[53] Jha, D. K., Kant, T., Singh, R. K., A critical review of recent research on functionally graded plates, Composite Structures 96 (2013) 833-849. https://doi.org/10.1016/j.compstruct.2012.09.001

[54] Kadoli, R., Akhtar, K., Ganesan, N., Static analysis of functionally graded beams using higher order shear deformation theory, Applied Mathamatical Modelling 32 (2008) 2509-2525. https://doi.org/10.1016/j.apm.2007.09.015

[55] Kahya, V., Turan, M., Finite element model for vibration and buckling of functionally graded beams based on the first order shear deformation theory, Composites Part-B 109 (2017) 108-115. https://doi.org/10.1016/j.compositesb.2016.10.039

[56] Kant, T., Gupta, A., A finite element model for higher order shears deformable beam theory, Journal of Sound and Vibration 125 (2) (1988) 193-202. https://doi.org/10.1016/0022-460X(88)90278-7

[57] Khalifa, Z., Hadji, L., Daouadji, T. H., Bourada, M., Buckling response with stretching effect of carbon nanotube-reinforced composite beams resting on elastic foundation, Structural Engineering and Mechanics 67 (2) (2018) 125-130.

[58] Kim, J., Paulino, G. H., Finite element evaluation of mixed mode stress intensity factors in functionally graded materials, International Journal for Numerical Methods in Engineering 53 (2002) 1903-1935. https://doi.org/10.1002/nme.364

[59] Koizumi, M., FGM activities in Japan, Composites Part-B 28 (1997) 1-4. https://doi.org/10.1016/S1359-8368(96)00016-9

[60] Koizumi, M., The concept of FGM. Ceramic transactions. Functionally Gradient Materials 34 (1993) 3-10.

[61] Li, S. R., Batra, R. C., Relations between buckling loads of functionally graded Timoshenko and homogeneous Euler-Bernoulli beams, Composite Structures 95 (2013) 5-9. https://doi.org/10.1016/j.compstruct.2012.07.027

[62] Li, X.F., Wang, B.L., Han, J.C., A higher-order theory for static and dynamic analyses of functionally graded beams, Archive of Applied Mechanics 80 (2010) 1197-1212. https://doi.org/10.1007/s00419-010-0435-6

[63] Ma, L. S., Lee, D. W., A further discussion of nonlinear mechanical behavior for FGM beams under inplane thermal loading, Composite Structures 93 (2011) 831-842. https://doi.org/10.1016/j.compstruct.2010.07.011

[64] Ma, L. S., Lee, D. W., Exact solutions for nonlinear static responses of a shear deformable FGM beam under an inplane thermal loading, European Journal of Mechanics-A/Solids 31 (2012) 13-20. https://doi.org/10.1016/j.euromechsol.2011.06.016

[65] Ma, L. S., Wang, T. J., Nonlinear bending and post-buckling of a functionally graded circular plate under mechanical and thermal loadings, International Journal of Solids and Structure 40 (13) (2003) 3311-3330. https://doi.org/10.1016/S0020-7683(03)00118-5

[66] Megharbel, A. E., A theoretical analysis of functionally graded beam under thermal loading, World Journal of Engineering and Technology 4 (2016) 437-449. https://doi.org/10.4236/wjet.2016.43044

[67] Menaa, R., Tounsi, A., Mouaici, F., Mechab, I., Zidi, M., Bedia, E. A. A., Analytical solutions for static shear correction factor of functionally graded rectangular beams, Mechanics of Advanced Materials and Structure 19 (2012) 641-652. https://doi.org/10.1080/15376494.2011.581409

[68] Mohanty, S. C., Dash, R. R., Rout, T., Static and dynamic stability analysis of a functionally graded Timoshenko beam, International Journal of Structural Stability and Dynamics 12 (4) (2012) 1-33. https://doi.org/10.1142/S0219455412500253

[69] Muller, E., Drasar, C., Schilz, J., Kaysser, W. A., Functionally graded materials for sensor and energy applications, Materials Science and Engineering 362 (2003) 17-39.

https://doi.org/10.1016/S0921-5093(03)00581-1 
[70] Naik, N.S., Sayyad, A.S., 1-D analysis of laminated composite and sandwich plates using a new fifthorder plate theory, Latin American Journal of Solids and Structures 15 (2018) 1-17. https://doi.org/10.1590/1679-78253973

[71] Naik, N. S., Sayyad, A. S., 2D analysis of laminated composite and sandwich plates using new fifth order theory, Latin American Journal of Solids and Structures 15 (9) (2018) 114-125. https://doi.org/10.1590/1679-78254834

[72] Naik, N.S., Sayyad, A.S., An accurate computational model for thermal analysis of laminated composite and sandwich plates, Journal of Thermal Stresses 42 (5) (2019) 559-579. https://doi.org/10.1080/01495739.2018.1522986

[73] Naik, N. S., Sayyad, A. S., New displacement for accurate prediction of transverse shear stresses in laminated and sandwich rectangular plates, ASCE Journal of Aerospace Engineering 32 (5) (2019) 1-12. https://doi.org/10.1061/(ASCE)AS.1943-5525.0001074

[74] Nguyen, T. K., Vo, T. P., Thai, H. T., Static and free vibration of axially loaded functionally graded beams based on the firstorder shear deformation theory, Composites Part-B 55 (2013) 147-157. https://doi.org/10.1016/j.compositesb.2013.06.011

[75] Nirmala, K., Upadhyay, P. C., Prucz, J., Lyons, D., Thermoelastic stresses in composite beams with functionally graded layer, Journal of Reinforced Plastics and Composites 2 (12) (2006) 1241-1254. https://doi.org/10.1177/0731684406059787

[76] Pande, V.B., Parashar, S. K., Static bending and dynamic analysis of functionally graded piezoelectric beam subjected to electromechanical loads, Proceedings of the Institution of Mechanical Engineers, Part C: Mechanical Engineering Science 230 (19) (2016) 3457-3469. https://doi.org/10.1177/0954406215596359

[77] Pendhari, S. S., Kant, T., Desai, Y. M., Subbaiah, C. V., On deformation of functionally graded narrow beams under transverse loads, International Journal of Mechanics and Materials in Design 6 (2010) 269-282. https://doi.org/10.1007/s10999-010-9136-0

[78] Rabahi, A., Rabia, B., Daouadji, T.H., Belkacem, A., Abbes, F., Elastic analysis of interfacial stresses in prestressed PFGM-RC hybrid beams, Advances in Materials Research 7 (2) (2018) 83-103.

[79] Reddy, J. N., A simple higher order theory for laminated composite plates, Journal of Applied Mechanics 51 (1984) 745-752. https://doi.org/10.1115/1.3167719

[80] Sankar, B. V., An elasticity solution for functionally graded beam, Composites Science and Technology 61 (2001) 689-696. https://doi.org/10.1016/S0266-3538(01)00007-0

[81] Sankar, B. V., Tzeng, J. T., Thermal stresses in functionally graded beams, AIAA Journal 40 (6) (2002) 1-5. https://doi.org/10.2514/2.1775

[82] Sator, L., Sladek, V., Sladek, J., Bending of FGM plates under thermal load: classical thermo-elasticity analysis by a meshless method, Composites Part-B 146 (2018) 176-188. https://doi.org/10.1016/j.compositesb.2018.04.004

[83] Sayyad, A.S., Ghugal, Y. M., A unified shear deformation theory for the bending of isotropic, functionally graded, laminated and sandwich beams and plates, International Journal of Applied Mechanics 9 (1) (2017) 1-36.

[84] Sayyad, A. S., Ghugal, Y.M., Analytical solutions for bending, buckling, and vibration analyses of exponential functionally graded higher order beams, Asian Journal of Civil Engineering 19 (5) (2018) 607-623. https://doi.org/10.1007/s42107-018-0046-z

[85] Sayyad, A.S., Ghugal, Y. M., Bending, buckling and free vibration of laminated composite and sandwich beams: A critical review of literature, Composite Structures 171 (2017) 486-504. https://doi.org/10.1016/j.compstruct.2017.03.053

[86] Sayyad, A. S., Ghugal, Y. M., Effects of non-linear hygro-thermo-mechanical loading on bending of FGM rectangular plates resting on two-parameter elastic foundation using four-unknown plate theory, Journal of Thermal Stresses 42 (2) (2018) 213-232.

https://doi.org/10.1080/01495739.2018.1469962 
[87] Sayyad, A. S., Ghugal, Y. M., Modeling and analysis of functionally graded sandwich: A review, Mechanics of Advanced Materials and Structures 26 (21) (2019) 1776-1795. https://doi.org/10.1080/15376494.2018.1447178

[88] Sayyad, A. S., Ghugal, Y. M., On the free vibration analysis of laminated composite and sandwich plates: A review of recent literature with some numerical results, Composite Structures 129 (2015) 177-201. https://doi.org/10.1016/j.compstruct.2015.04.007

[89] Shen, H. S., Nonlinear bending response of functionally graded plates subjected to transverse loads and in thermal environments, International Journal of Mechanical Sciences 44 (2002) 561-584. https://doi.org/10.1016/S0020-7403(01)00103-5

[90] Swaminathan, K., Naveenkumar, D. T., Zenkour, A. M., Carrera, E., Stress, vibration and buckling analyses of FGM plates - A state of the art review, Composite Structures 120 (2015) 10-31. https://doi.org/10.1016/j.compstruct.2014.09.070

[91] Swaminathan, K., Sangeetha, D. M., Thermal analysis of FGM plates - A critical review of various modeling techniques and solution methods, Composite Structures 160 (2017) 43-60. https://doi.org/10.1016/j.compstruct.2016.10.047

[92] Thai, H. T., Vo, T. P., Bending and free vibration of functionally graded beams using various higher order shear deformation beam theories, International Journal of Mechanical Sciences 62 (2012) 57-66. https://doi.org/10.1016/j.ijmecsci.2012.05.014

[93] Toudehdehghan, A., Rahman, M.M., Tarlochan, F., Mechanical and thermal analysis of classical functionally graded coated beam, E3S Web of Conferences 34 (2018) 01033. https://doi.org/10.1051/e3sconf/20183401033

[94] Xu, Y., Yu, T., Zhou, D., Two-dimensional elasticity solution for bending of functionally graded beams with variable thickness, Meccanica 49 (10) (2014) 2479-2489. https://doi.org/10.1007/s11012-014-9958-1

[95] Ying, J., Lu, C. F., Chen, W. Q., Two-dimensional elasticity solutions for functionally graded beams resting on elastic foundations, Composite Structures 84 (2008) 209-219. https://doi.org/10.1016/j.compstruct.2007.07.004

[96] Zenkour, A. M., Transverse shear and normal deformation theory for bending analysis of laminated and sandwich elastic beams, Mechanics of Advanced Materials and Structures 6 (1999) 267-283. https://doi.org/10.1080/107594199305566

[97] Zenkour, A. M., Allam, M.N., Radwan, A. F., Effects of transverse shear and normal strains on FG plates resting on elastic foundations under hygro-thermo-mechanical loading, International Journal of Applied Mechanics 6 (5) (2014) 1-26.

[98] Zenkour, A. M., Radwan, A. F., Hygro-thermo-mechanical buckling of FGM plates resting on elastic foundations using a quasi-3D model, International Journal of Computational Methods in Engineering Science and Mechanics (2019) 1-15. https://doi.org/10.1080/15502287.2019.1568618

[99] Zhong, Z., Yu, T., Analytical solution of a cantilever functionally graded beam, Composites Science and Technology 67 (2007) 481-488. https://doi.org/10.1016/j.compscitech.2006.08.023

[100] Zhou, H. M., Zhang, X. M., Wang, Z. Y., Thermal analysis of 2D FGM beam subjected to thermal loading using meshless weighted least-square method, Mathematical Problems in Engineering (2019) 2541707. https://doi.org/10.1155/2019/2541707

[101] Zidi, M., Taunsi, A., Hauari, M., Bedia, E., Beg, O. A., Bending analysis of an FGM plates under hygro-thermo-mechanical loading using a four variable refined plate theory, Aerospace Science and Technology 14 (2014) 24-34. https://doi.org/10.1016/j.ast.2014.02.001 